\title{
Light-triggered Enantioselective Organocatalytic Mannich-type Reaction
}

\author{
Hamish B. Hepburna \\ Giandomenico Magagnano ${ }^{a}$ \\ Paolo Melchiorre*a,b \\ ${ }^{a}$ ICIQ - Institute of Chemical Research of Catalonia \\ Barcelona Institute of Science and Technology, \\ Països Catalans 16, 43007 - Tarragona, Spain \\ ${ }^{b}$ ICREA - Pg. LLuís Companys 23, 08010 - Barcelona, Spain \\ pmelchiorre@iciq.es
}

Dedicated to Professor Dieter Enders on the occasion of his 70th birthday

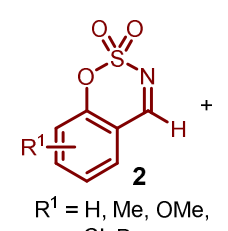

$\mathrm{Cl}, \mathrm{Br}$<smiles>[R]Cc1ccccc1C(N)=O</smiles>
$R^{2}=H, M e$ $(D H Q D)_{2} P H A L$
$(20 \mathrm{~mol} \%)$

black LED

cyclohexane

ambient temperature<smiles>[R]C(c1ccccc1)C1NS(=O)(=O)Oc2ccccc21</smiles>

$\mathrm{R}^{1}$<smiles>[R]C=c1ccccc1=C(O)Br</smiles>

2

via photoenolization

Abstract Disclosed herein is a photochemical organocatalytic strategy for the direct enantioselective Mannich-type reaction of 2-alkyl-benzophenones and cyclic imines. The chemistry exploits the light-triggered enolization of 2-alkyl-benzophenones to generate transient hydroxy-o-quinodinomethanes. These fleeting intermediates can be stereoselectively intercepted by imines upon activation with a chiral organic catalyst, derived from natural cinchona alkaloids. The developed method uses mild conditions, simple sources of illumination and easily available substrates and catalysts, affording enantioenriched chiral amines that are difficult to synthesize by other approaches.

Key words: enantioselective catalysis, organocatalysis, Mannich-type reaction, photochemistry, synthetic methods.

The possibility of generating highly reactive hydroxyl-o-quinodimethanes $\mathbf{A}$ through the photoexcitation of 2-alkyl benzophenones $\mathbf{1}$ has been reported as far back as $1961 .{ }^{1}$ Transient intermediates $\mathbf{A}^{2}$ can serve as suitable dienes for a range of [4+2] cycloadditions with electron-poor alkenes $\mathbf{2}$ (Figure 1a). ${ }^{3}$ The resulting Diels-Alder processes afford synthetically valuable benzannulated carbocyclic products 3. The mechanism of formation of $\mathbf{A}$ has been well-studied and characterized (Figure 1b). ${ }^{4}$ Irradiation of the 2-alkyl benzophenone $\mathbf{1}$ triggers the formation of a singlet excited state $S_{1}-\mathbf{B}$ that, upon intersystem crossing, decays to a triplet state $\mathrm{T}_{1}$-B. Following 1,5-hydrogen transfer, the diradical intermediate $(Z)$-C is generated, which then undergoes rotation to afford the highly reactive enol $(E)$-A. Chemical trapping of $\mathbf{A}$ by a dienophile $\mathbf{2}$ provides straightforward access to stereochemically dense cyclic derivatives $\mathbf{3}$.

The photoenolization/Diels-Alder sequence, in racemic fashion, has been extensively used by chemists.2,5 However, developing an enantioselective catalytic variant has proven a difficult target. Asymmetric catalytic approaches are greatly complicated by the high reactivity and fleeting nature of the photoenols $\mathbf{A}$, which make it difficult for a chiral catalyst to channel the process through a stereocontrolled pathway. One effective asymmetric method used a stoichiometric amount of a chiral complexing agent to selectively bind a purposely designed 2-alkyl carbonyl compound. ${ }^{6}$ But methods that use substoichiometric chiral catalysts remained unprecedented until recently, when our research group reported an organocatalytic strategy for successfully trapping $\mathbf{A}$ in a stereoselective fashion. ${ }^{7}$ Our approach relied on the use of a chiral organic catalyst which could effectively activate the dienophilic maleimide D (Figure 1c). 


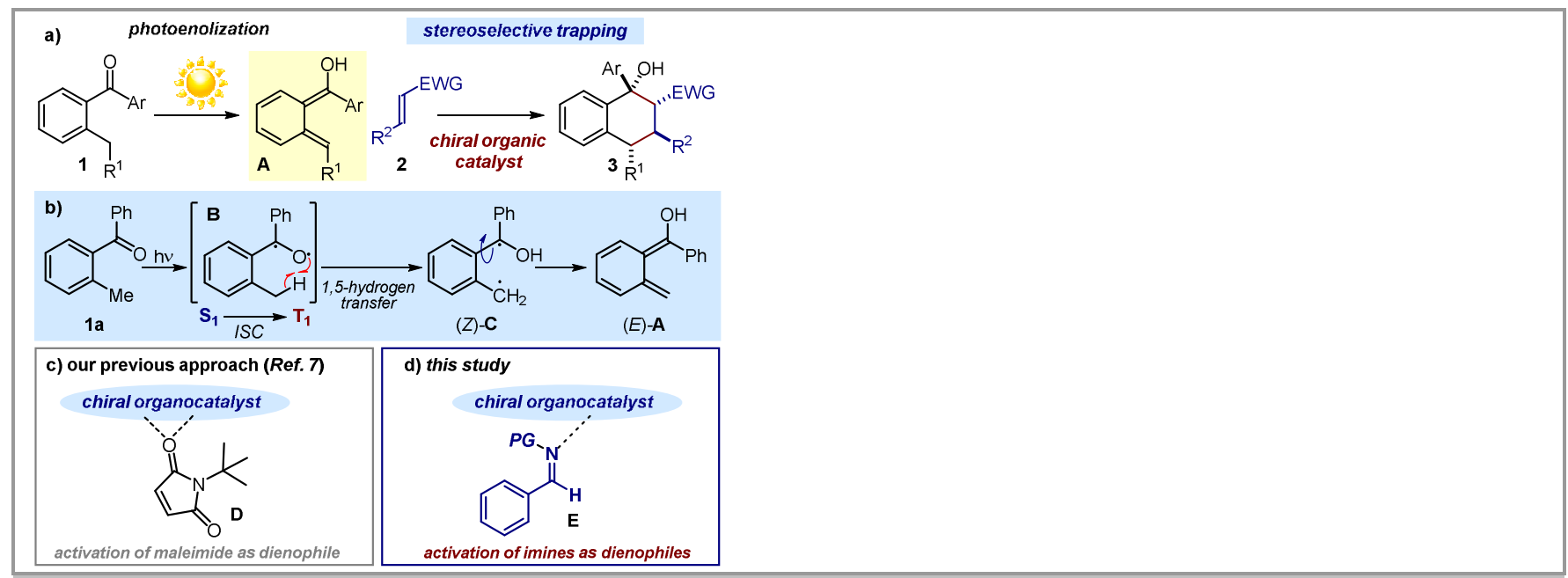

Figure 1 a) The classical photoenolization/Diels-Alder strategy; EWG = electron withdrawing group. b) The photoenolization mechanism of 2-methylbenzophenone 1a; ISC: intersystem crossing. c) Our precedent organocatalytic approach for activating maleimide $\mathbf{B}$ toward the stereoselective trap of hydroxy-o-quinodimethanes $\mathbf{A}$. d) The proposed trap of photoenol $\mathbf{A}$ with an imine $\mathbf{C}$ activated by a chiral organic catalyst; PG: protecting group.

Building upon this precedent, we wondered whether organocatalysis ${ }^{8}$ could offer other effective tools to stereoselectively trap photogenerated hydroxy-o-quinodimethanes A with different dienophiles. Specifically, we considered imines $\mathbf{E}$ as suitable candidates for the interception of $\mathbf{A}$ (Figure 1d). Although no literature precedent exists for even the racemic version of such transformation, we were motivated by the notions that imines are primed to organocatalytic activations ${ }^{9}$ and can generally participate in hetero-Diels Alder processes..$^{10}$ Herein, we document how this idea was translated to experimental reality.

We started out our investigations studying the reaction between the commercially available 2-methylbenzophenone 1a and a variety of $N$-protected benzaldimines 2 . These processes were conducted in the presence of 20 mol\% of the phosphoric acid $\mathbf{5 a}^{11 \text { a }}$ or the cinchonabased thiourea $\mathbf{5 b}^{11 \mathrm{~b}}$ (Figure 2), two chiral organic catalysts with an established ability to activate imine substrates while inducing high level of stereoselectivity. ${ }^{11}$ We also tested the catalytic profile of $\mathbf{5 c}$, since it provided the best results in our previous study on the stereocontrolled trapping of photoenol A with maleimides of type D (Figure 1c). ${ }^{7}$ The experiments were performed over 14 hours, in toluene, at ambient temperature, and under irradiation by a single black-light-emitting diode (black LED, $\lambda_{\max }=365 \mathrm{~nm}$ ).

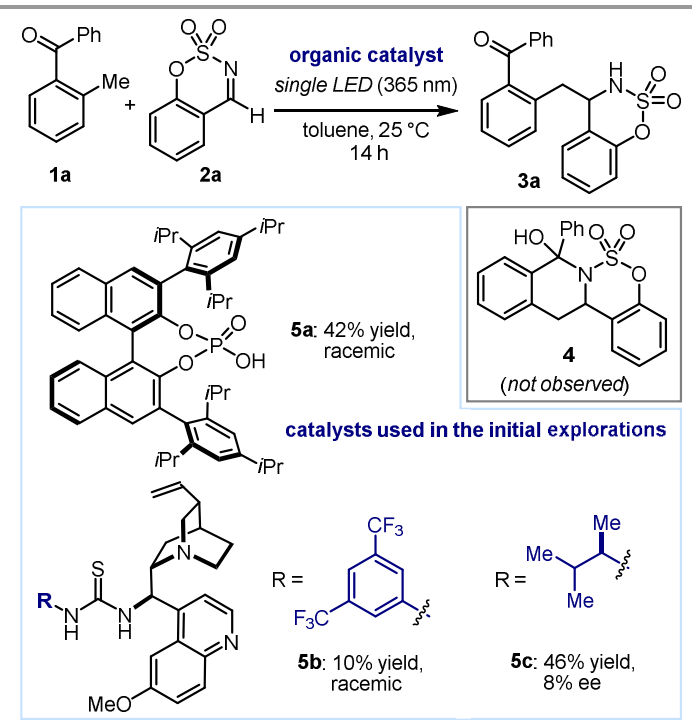

Figure 2 Initial explorations aimed at identifying the model reaction and a suitable imine substrate.

Benzaldimines bearing commonly used $N$-protecting groups, including $N$-Boc, $N$-tosyl, and $N$-PMP imines (Boc = tert-butyloxycarbonyl; tosyl = toluenesulfonyl; PMP = p-methoxyphenyl), did not lead to the formation of any product, and were recovered untouched. The lack of reactivity of linear imines prompted us to evaluate the behavior of benzoxathiazine-2,2-dioxide $\mathbf{2 a}$. Indeed, it has been recently reported that the cyclic nature of $\mathbf{2 a}$, by constraining the $\mathrm{C}=\mathrm{N}$ bond in a $\mathrm{Z}$ geometry, makes it a highly reactive substrate prone to undergo nucleophilic additions ${ }^{12}$ and hetero-Diels-Alder reactions. ${ }^{13}$ This reactivity profile provides a rationale for the capability of $\mathbf{2 a}$, in the presence of catalyst $\mathbf{5 a - c}$, to successfully trap the fleeting hydroxy-o-quinodimethane A, generated from 1a (Figure 2). These observations come about with an unanticipated reactivity, since an unconventional formal Mannich-type reaction of the photoenol with 2a was observed instead of the expected [4+2] manifold. The light-triggered process led to the exclusive formation of the adduct 3a, while no traces of the cyclic product 4 were detected. 
Having identified a suitable substrate combination, we next focused on obtaining a high level of stereocontrol, since the light-triggered Mannich-type processes catalyzed by $\mathbf{5 a}, \mathbf{5 b}$, and $\mathbf{5} \mathbf{c}$ offered minimal level of enantioinduction, if any (product $\mathbf{3 a}$ formed as a racemate or with $8 \%$ ee, Figure 2). We undertook the evaluation of a large and diverse set of chiral organic catalyst structures (full details can be found in the Supporting Information), which identified the dimeric cinchona alkaloid derivative (DHQ) ${ }_{2}$ PHAL 6a, commonly employed as a ligand for Sharpless asymmetric dihydroxylation, ${ }^{14}$ as a promising candidate (Table 1). Indeed, the commercially available catalyst $\mathbf{6 a}$ afforded the amine product 3a in 58\% yield and with an enantiomeric excess as high as $60 \%$ in toluene (entry 1). Evaluation of the standard reaction parameters revealed that the use of a non-polar solvent such as cyclohexane increased the level of stereoselectivity up to $72 \%$ ee (entry 2).

Table 1: Optimization Studies ${ }^{a}$

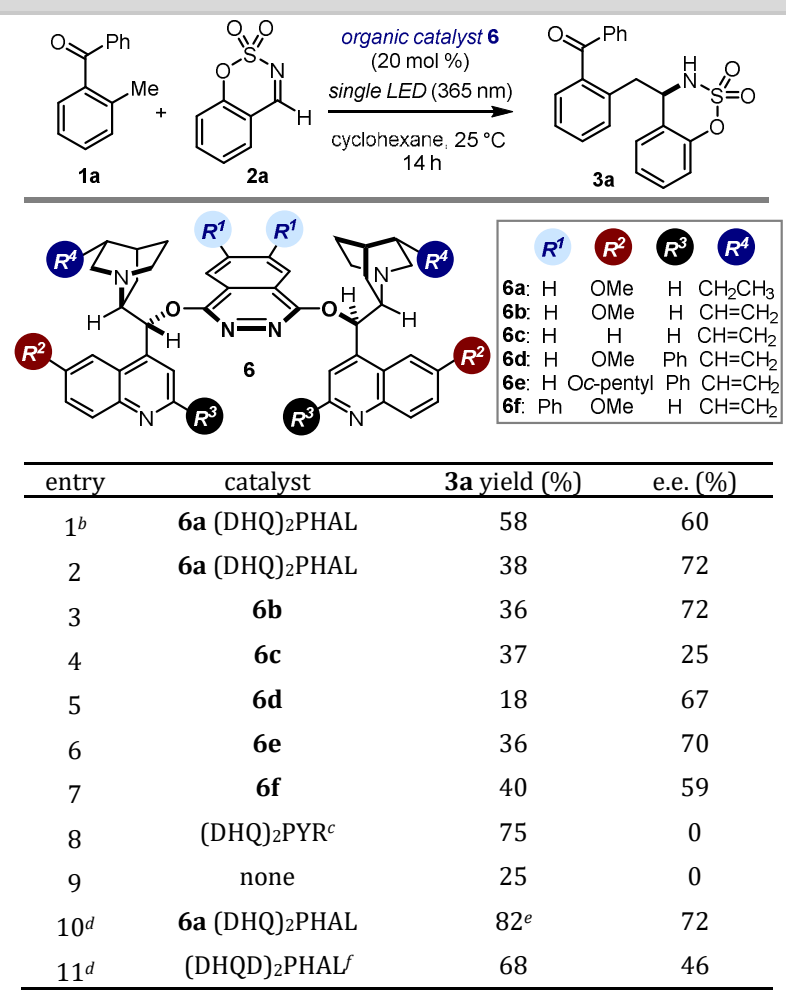

a Reactions performed using $20 \mathrm{~mol} \%$ of catalyst $\mathbf{6}, 2$ equiv. of $1 \mathrm{a}$ and $[\mathbf{2} \mathrm{a}]_{0}=0.025 \mathrm{M}$ in cyclohexane; yield determined by ${ }^{1} \mathrm{H}$ NMR analysis of the crude mixture using trichloroethylene as the internal standard; ee determined by UPC ${ }^{2}$ analysis on a chiral stationary phase. ${ }^{b}$ Reaction performed in toluene. ${ }^{\mathrm{c}}$ The structure of (DHQ) ${ }_{2} \mathrm{PYR}$ catalyst,

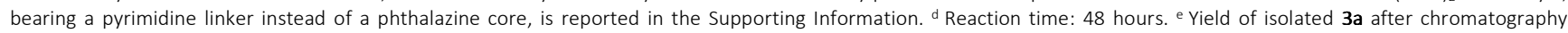
purification. ${ }^{\mathrm{f}}$ Reaction performed using the pseudoenantiomeric catalyst (DHQD) ${ }_{2} \mathrm{PHAL}$ to furnish ent-3a.

We then wondered if the stereoselectivity of the reaction could be improved by structurally modifying the dimeric cinchona scaffold of catalyst 6a. Unfortunately, extensive modifications of both the phthalazine linker and the cinchona moiety at a variety of sites did not bring about any improvement in enantioinduction (a representative selection is shown in Table 1, further details can be found in the Supporting Information). Still, these studies established the key role played by both the methoxy group on the quinoline ring ( $\mathrm{R}^{2}$ group in Table 1 , compare catalysts $\mathbf{6 a}$ and $\mathbf{6 c}$ in entries 2 and 4 ) and the two contiguous nitrogen atoms within the phthalazine linker (compare catalysts $\mathbf{6 a}$ and (DHQ) 2 PYR in entries 2 and 8) in dictating the stereoselectivity.

A final cycle of optimization using the commercial catalyst (DHQ) ${ }_{2} \mathrm{PHAL} 6$ a revealed that the adduct 3a could be isolated with high chemical yield and with $72 \%$ ee when extending the reaction time to 48 hours (entry 10, 82\% yield). This level of enantioselectivity is striking when considering the substantial rate of background reaction observed in the absence of catalyst (entry 9). ${ }^{15,16}$ The use of the pseudoenantiomeric (DHQD) 2 PHAL catalyst, derived from hydroquinidine, provided access to the antipode of the Mannich-type product ent-3a, albeit with a reduced enantiomeric excess (46\% ee, entry 11$)$.

We next sought to explore the scope of both substrates in the asymmetric organocatalytic photenolization/Mannich-type reaction sequence, using the reaction conditions detailed in entry 10 of Table 1. As shown in Scheme 1, there appears to be significant tolerance for structural and electronic variations of the benzophenone derivatives 1 to enable access to a variety of amine products $\mathbf{3}$. Substitution on the non-enolizable aromatic ring of $\mathbf{1}$ with both electron-donating and electron-withdrawing groups is well tolerated. The reaction proceeds well with substrates bearing substituents at the meta- and para- positions (products $\mathbf{3 b}$ - $\mathbf{3 h}$ ), while an ortho-substitution pattern negatively affects the stereoselectivity (product 3i). The presence of a substituent at the benzylic $\mathrm{R}^{2}$ position of $\mathbf{1}$ brings about the formation of two contiguous stereogenic centers (product 3j), albeit with a low diastereoselectivity. Substitution of the enolizable phenyl ring led to a decreased stereocontrol (product 3k) while providing a high chemical yield. Not all 2-methylbenzophenones $\mathbf{1}$ are suitable for the reaction; substrates featuring extended $\pi$-systems $(\mathbf{1 1}, \mathbf{1 m})$ or sterically encumbered substitution patterns $(\mathbf{1 n})$ remained unreacted. Crystals from compound 3e were suitable for X-ray crystallographic analysis, ${ }^{17}$ which established the stereochemical outcome 
of the Mannich-type reaction and the absolute configuration of the newly-formed stereogenic center. From a synthetic perspective, it is interesting that, although the amine products $\mathbf{3}$ are generally obtained in moderate enantioselectivity, the optical purity can be easily increased by simple crystallization, as demonstrated for compound 3d (96\% ee).

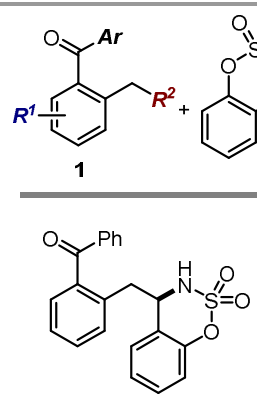

3a: $82 \%$ yield, $72 \%$ ee<smiles>O=C(c1ccc(Br)cc1)c1ccccc1CC1NS(=O)(=O)Oc2ccccc21</smiles>

3d: $79 \%$ yield, $77 \%$ ee $(96 \% \text { ee })^{a}$<smiles>COc1cccc(C(=O)c2ccccc2CC2NS(=O)(=O)Oc3ccccc32)c1</smiles>

3f: $73 \%$ yield, $69 \%$ ee

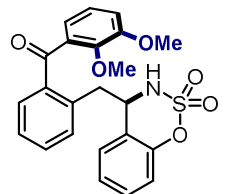

3i: $77 \%$ yield, $39 \%$ ee<smiles>COc1ccc(C(=O)c2ccccc2CC2NS(=O)(=O)Oc3ccccc32)cc1</smiles>

3b: $66 \%$ yield, $73 \%$ ee
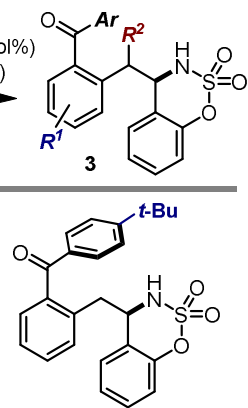

3c: $74 \%$ yield, $77 \%$ ee

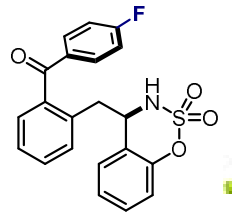

3e: $82 \%$ yield, $75 \%$ ee

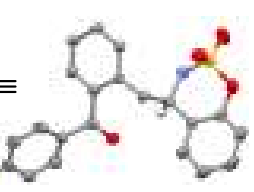

$C C D C 1497811$<smiles>O=C(c1cccc(Cl)c1)c1ccccc1CC1NS(=O)(=O)Oc2ccccc21</smiles>

$3 g, 70 \%$ yield, $72 \%$ ee<smiles>O=C(c1cccc(Br)c1)c1ccccc1CC1NS(=O)(=O)Oc2ccccc21</smiles>

3h: $74 \%$ yield, $71 \%$ ee<smiles>C[C@H](c1ccccc1C(=O)P)[C@@H]1NS(=O)(=O)Oc2ccccc21</smiles>

3j: $66 \%$ yield, $1.3: 1 \mathrm{~d}$ $68 \%$ ee/ $60 \%$ ee

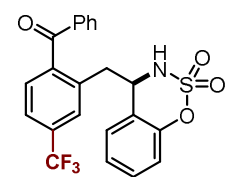

3k: $84 \%$ yield, $37 \%$ ee<smiles>Cc1ccccc1C(=O)c1ccc2ccccc2c1</smiles>

Scheme 1: Survey of the benzophenones 1 that can participate in the photochemical organocatalytic Mannich-type reaction. Reactions performed using 100 umol of $2 \mathrm{a}, 50 \mathrm{\mu mol}$ of 1 and $20 \mathrm{~mol} \%$ of catalyst $6 \mathbf{a}$ in cyclohexane $(2 \mathrm{~mL})$. Yields and the enantiomeric excesses of the isolated products 3 are indicated below each entry (average of two runs per substrate). ${ }^{a}$ Ee value obtained after a single crystallization.

As for the cyclic imines $\mathbf{2}$ that can participate in the light-triggered organocatalytic Mannich-type reaction (Scheme 2), different alkyl substituents are well tolerated at the ortho-, meta-, and para- positions (with respect to the oxygen), affording products 7a-d in good yields and moderate enantioselectivity. The presence of an electron-donating group negatively influences the reactivity of the imine, resulting in a lower isolated yield while maintaining a good stereoselectivity (product 7e). Electron-withdrawing groups provide the products $\mathbf{7 f - h}$ in good yields but with a reduced enantioselectivity. 


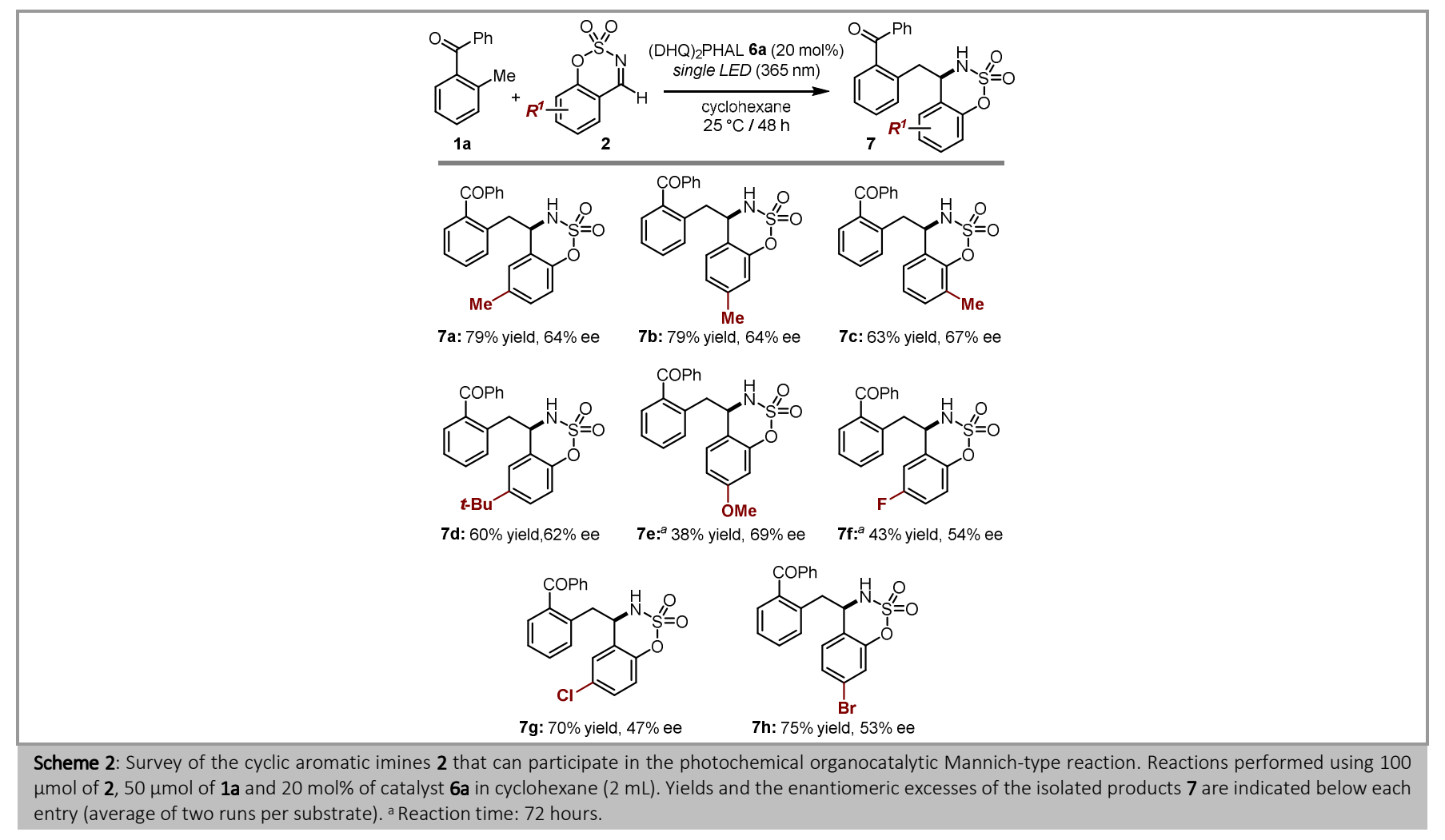

Mechanistically, several aspects of this photo-mediated process deserve comment. The most peculiar trait is that no traces of the expected cycloaddition adducts 4 (see Figure 2) were detected during our studies. Given the well-established tendency of both photogenerated hydroxy-o-quinodimethanes ${ }^{2,3,5}$ and cyclic imines of type $2^{13}$ to engage in hetero-Diels-Alder pathways, the occurrence of a [4+2]cycloaddition manifold, followed by the spontaneous collapse of the hemiaminal $\mathbf{4}$, is likely. A similar hetero-Diels-Alder/ring-opening sequence has been recently proposed (and supported by calculations) as the underlying mechanism of the light-driven carboxylation of ketones $\mathbf{1}$ proceeding via the trapping of the photoenol $\mathbf{A}$ with $\mathrm{CO}_{2}{ }^{18}$

As for the mechanism of stereocontrol, we initially thought that (DHQ) 2 PHAL 6a could interact with the transient photoenol (E)-A. This idea was motivated by our precedent studies ${ }^{7}$ demonstrating that the quinuclidine core within the cinchona-based catalyst $\mathbf{5 c}$ could reduce the formation of the photoenol derived from 1a by means of an electron transfer mechanism. To evaluate whether the cinchona moiety in 6a could participate in a similar pathway, we conducted laser flash photolysis studies. These investigations, detailed in the Supporting Information, showed that the absorption at $450 \mathrm{~nm}$ of the transient photoenol A (half lifetime $7 \mathrm{~ms}$ in toluene), generated upon laser excitation of $\mathbf{1 a}$, was not significantly affected by the presence of $\mathbf{6 a} .{ }^{16}$ Overall, our observations suggest that the chiral catalyst $\mathbf{6 a}$ controls the stereochemical outcome of the reaction by solely interacting with the imine substrate $1 .{ }^{19}$ Additional studies are underway in our laboratories to shed further light upon the mechanism governing the stereoinduction of the light-triggered Mannich-type reaction.

In conclusion, we have documented the possibility to stereoselectively trap hydroxyl-o-quinodimethane A, generated upon light excitation of 2-alkyl benzophenones $\mathbf{1}$, with cyclic imines $\mathbf{2}$. This process, which has no precedents in the racemic regime too, leads to the formation of chiral amines $\mathbf{3}$ via a formal Mannich-type reaction manifold. The developed method uses mild conditions, simple sources of illumination and the commercially available (DHQ) 2 PHAL as catalyst, which can induce a good level of stereoselectivity. Further studies are ongoing to provide a deeper mechanistic understanding while identifying additional dieneophiles that may serve to stereoselectively trap the photoenol A.

\section{The experimental section has no title; please leave this line here.}

Commercial grade reagents and solvents were purchased at the highest commercial quality and used without further purification, unless otherwise stated. Cyclohexane was purchased from Sigma Aldrich and was sparged with argon for 30 minute before use. (DHQ) 2 PHAL 6a was purchased from Sigma Aldrich. Cyclic imines $\mathbf{2}^{20}$ and benzophenones ${ }^{7}$ were prepared according to procedures reported in the literature. Chromatographic purification of products was accomplished using force-flow chromatography (FC) on silica gel (35-70 mesh). For thin layer chromatography (TLC) analysis throughout this work, Merck precoated TLC plates (silica gel $60 \mathrm{GF}_{254}, 0.25 \mathrm{~mm}$ ) were used, using UV light as the visualising agent.

The NMR spectra were recorded at $500 \mathrm{MHz}$ for ${ }^{1} \mathrm{H}$ or at $125 \mathrm{MHz}$ for ${ }^{13} \mathrm{C}$, respectively. The chemical shifts $(\delta)$ for ${ }^{1} \mathrm{H}$ and ${ }^{13} \mathrm{C}$ are given in ppm relative to residual signals of the solvents $\left(\mathrm{CHCl}_{3} @ 7.27 \mathrm{ppm}{ }^{1} \mathrm{H}\right.$ NMR, $\left.77.00 \mathrm{ppm}{ }^{13} \mathrm{C} \mathrm{NMR}\right)$. Coupling constants are given in Hz. The following abbreviations are used to indicate the multiplicity: s, singlet; d, doublet; t, triplet; q, quartet; m, multiplet; br s, broad signal. High-resolution mass spectra (HRMS) were obtained from the ICIQ High Resolution Mass Spectrometry Unit on Waters GCT gas chromatograph coupled time-of-flight mass spectrometer (GC/MS-TOF) with electron ionization (EI) or MicroTOF II (Bruker Daltonics): HPLC-MS-TOF (ESI). X-ray data were obtained from the ICIQ X-Ray Unit using a Bruker-Nonius diffractometer equipped with an APPEX 24 K CCD area detector. Optical rotations were measured on a Polarimeter Jasco P-1030 and are reported as follows: $[\alpha]_{\mathrm{D}} \mathrm{rt}$ (c in g per $100 \mathrm{~mL}$, solvent). FT-IR measurements were carried out on an Agilent Technologies Cary 630 FTIR spectrometer. Melting points were measured on a Mettler Toledo MP70 melting point system and are uncorrected. Studies with millisecond transient absorption spectroscopy (TAS) were performed using an excitation source of Nd:YAG (neodymium-doped yttrium aluminium garnet) tuned with an optical parametric oscillator (OPO) from 
Opolette as a pump source. This laser produces $6 \mathrm{~ns}$ pulses of $1 \mathrm{~mJ}$ at a wavelength of $355 \mathrm{~nm}$. The system is completed with two monochromators with double grating at the VIS an IR, and a digital recorder DSP-DAU from RAMDSP. A photodetector amplifiers and a software control complete the TAS system.

Determination of Enantiomeric Purity: UPC ${ }^{2}$ and HPLC analyses on chiral stationary phase were performed on a Waters Acquity and an Aglient 1200 series instrument, respectively. Traces were compared to racemic samples prepared by running the reaction in the absence of catalyst.

Light Source: The light source used for illuminating the reaction vessel consisted of a single black LED (3.6 W, EOLD-365-525 LED, UV, 5 mm, $\lambda$ max= 365 $\mathrm{nm}$ ) produced by OSA OPTO Light and purchased from Farnell (http://www.farnell.com/).

General Procedure for the light-triggered enantioselective organocatalytic Mannich-type reaction: A $5 \mathrm{~mL}$ glass vial was charged with the imine 2 $(50 \mu \mathrm{mol})$ and the catalyst (DHQ) 2 PHAL 6a $(10 \mu \mathrm{mol}, 0.2$ equiv., $7.8 \mathrm{mg})$. The vial was capped with a septum and purged with vacuum/argon cycles (x 3$)$. The benzophenone 2 ( 2 equiv.) was dissolved in degassed cyclohexane ( $2 \mathrm{~mL}$ ) and added to the vial. The vial was flushed with argon, closed with a Tefloncoated cap, sealed with parafilm and placed into a single black LED plate $\left(\lambda=365 \mathrm{~nm}\right.$, intensity $=6.5 \pm 0.5 \mathrm{~mW} / \mathrm{cm}^{2}$, as controlled by an external power supply; the setup is further detailed in the Supporting Information). Stirring at ambient temperature was maintained for 48 hours, and then the irradiation was stopped. The reaction was then diluted with methylene chloride $(1 \mathrm{~mL})$ and passed through a pad of silica. The volatiles were removed in vacuum and the residue was purified by flash column (FC) chromatography to give the amine product 3 in the stated yield and enantiomeric purity. The experiment was repeated and the results are an average of two runs.

Experimental Note A: when colleting the final amine products 3/7 after chromatography, in vacuo concentration should be made from a non-polar solution, such as hexane. This secures the resultant product to be obtained as a crystalline solid. If the final product is concentrated from a polar solution instead, such as $\mathrm{CH}_{2} \mathrm{Cl}_{2}$ or $\mathrm{CHCl}_{3}$, this would provide a gummy solid, which may need further mechanical handling to form a crystalline solid.

Experimental Note B: The amine products $3 / 7$ have generally a low solubility in non-polar solvent. To ensure consistent UPC ${ }^{2}$ analysis of the enantiomeric excess, it is necessary to dissolve the whole sample in a 7:3 hexane:iPrOH solution and then taking a aliquot for analysis.

\section{(R)-(2-((2,2-Dioxido-3,4-dihydrobenzo[e][1,2,3]oxathiazin-4-yl)methyl)phenyl)(phenyl)methanone (3a)}

The title compound 3a was prepared according to the general procedure using $\mathbf{2 a}(9.2 \mathrm{mg}, 50 \mu \mathrm{mol})$ and 2 -methylbenzophenone $\mathbf{1 a}(18 \mu \mathrm{L}, 100 \mu \mathrm{mol})$. The crude product was purified by FC (95:5 hexane/EtOAc) to afford amine 3a (15.5 mg, 82\%, average of two runs) as a white solid. $\mathrm{R}_{\mathrm{f}}=0.32$ (9:1 hexane/EtOAc); melting point $(\mathrm{mp})=55-57^{\circ} \mathrm{C}$ (upon recrystallized from hexane).

IR (ATR): 3103, 1636 (C=0), 1595, 1369, 1263, 1178, 1111, 938, 755, $705 \mathrm{~cm}^{-1}$.

1H NMR (500 MHz, CDCl $) \delta 7.81(2 \mathrm{H}, \mathrm{dt}, J=8.4,1.5 \mathrm{~Hz}, \mathrm{ArH}), 7.64(1 \mathrm{H}, \mathrm{ddt}, J=8.8,7.1,1.3 \mathrm{~Hz}, \operatorname{ArH}), 7.61-7.58(2 \mathrm{H}, \mathrm{m}, \mathrm{ArH}), 7.49(2 \mathrm{H}, \mathrm{ddt}, J=8.7,7.5,1.4$ $\mathrm{Hz}, \mathrm{ArH}), 7.44(1 \mathrm{H}, \mathrm{dt}, J=7.8,1.1 \mathrm{~Hz}, \mathrm{ArH}), 7.41-7.37(2 \mathrm{H}, \mathrm{m}, \mathrm{ArH}), 7.32(1 \mathrm{H}, \mathrm{dddd}, J=8.2,7.4,1.7,0.8 \mathrm{~Hz}, \mathrm{ArH}), 7.20(1 \mathrm{H}, \mathrm{td}, J=7.6,1.3 \mathrm{~Hz}, \mathrm{ArH}), 7.11(1 \mathrm{H}, \mathrm{d}$, $J=7.6 \mathrm{~Hz}, \mathrm{NH}), 7.06(1 \mathrm{H}, \mathrm{dd}, J=8.2,1.2 \mathrm{~Hz}, \mathrm{ArH}), 5.15(1 \mathrm{H}, \mathrm{ddd}, J=12.0,7.6,4.6 \mathrm{~Hz}, \mathrm{CHNH}), 3.53(1 \mathrm{H}, \mathrm{dd}, J=13.7,4.6 \mathrm{~Hz}, \mathrm{CH} 2 \mathrm{CHNH}), 3.25(1 \mathrm{H}, \mathrm{dd}, J=13.7$, $\left.11.5 \mathrm{~Hz}, \mathrm{CH}_{2} \mathrm{CHNH}\right)$.

${ }^{13}$ C NMR (125.6 MHz, CDCl 3 ) $\delta 199.4$ (C=0), 151.2 (C), $137.6(\mathrm{C}), 137.3(\mathrm{C}), 136.7$ (C), $133.8(\mathrm{CH}), 131.8(\mathrm{CH}), 131.2(\mathrm{CH}), 130.9(2 \times \mathrm{CH}), 130.7(\mathrm{CH}), 129.4$ $(\mathrm{CH}), 128.5(2 \times \mathrm{CH}), 126.5(\mathrm{CH}), 126.3(\mathrm{CH}), 125.1(\mathrm{CH}), 122.4(\mathrm{C}), 119.1(\mathrm{CH}), 58.7(\mathrm{CH}), 37.7\left(\mathrm{CH}_{2}\right)$.

HRMS (ESI) Exact mass calculated for $\mathrm{C}_{21} \mathrm{H}_{17} \mathrm{NO}_{4} \mathrm{SNa}[\mathrm{M}+\mathrm{Na}]^{+}: 402.0770$, found: 402.0776.

The enantiomeric excess was determined by UPC 2 analysis on a Waters Amy1 column, 100:0 $\mathrm{CO}_{2} / \mathrm{EtOH}$ to $60: 40 \mathrm{CO}_{2} / \mathrm{EtOH}$ for 9 minutes with curve of " 6 ", $\lambda=251 \mathrm{~nm}: \tau_{\text {minor }}=5.60 \mathrm{~min}, \tau_{\text {major }}=5.77 \mathrm{~min}(72 \%$ ee $) \cdot[\alpha]_{\mathrm{D}}^{28}=+18.9\left(\mathrm{c}=1.0, \mathrm{CHCl}_{3}, 72 \%\right.$ ee $)$.

\section{(R)-(2-((2,2-dioxido-3,4-dihydrobenzo[e][1,2,3]oxathiazin-4-yl)methyl)phenyl)(4-methoxyphenyl)methanone (3b)}

The title compound $\mathbf{3 b}$ was prepared according to the general procedure using $\mathbf{2 a}(9.2 \mathrm{mg}, 50 \mu$ mol) and (4-methoxyphenyl)(o-tolyl)methanone $\mathbf{1 b}(22.6$ $\mathrm{mg}, 100 \mu \mathrm{mol})$. The crude product was purified by FC ( $95: 5$ hexane/EtOAc) to afford amine $3 \mathbf{b}\left(13.5 \mathrm{mg}, 66 \%\right.$, average of two runs) as a white solid. $\mathrm{R}_{\mathrm{f}}=0.21$ (9:1 hexane/EtOAc); $\mathrm{mp}=50-52{ }^{\circ} \mathrm{C}$ (hexane).

IR (ATR): 2923, 1627 (C=0), 1590, 1485, 1453, 1419, 1373, 1257, 1169, $746 \mathrm{~cm}^{-1}$.

1H NMR (500 MHz, CDCl $)$ ) $8.63-7.55$ (2H, m, ArH), 7.45 (1H, dd, J= 7.5, 1.2 Hz, ArH), 7.42-7.35 (4H, m, ArH), 7.33 (1H, tdd, J= 7.4, 1.6, 0.7 Hz, ArH), 7.29 $(1 \mathrm{H}, \mathrm{dt}, J=7.7,1.3 \mathrm{~Hz}, \mathrm{ArH}), 7.22-7.16(2 \mathrm{H}, \mathrm{m}, \mathrm{ArH}), 7.12(1 \mathrm{H}, \mathrm{d}, J=7.4 \mathrm{~Hz}, \mathrm{NH}), 7.06(1 \mathrm{H}, \mathrm{dd}, J=8.3,1.3 \mathrm{~Hz}, \mathrm{ArH}), 5.15(1 \mathrm{H}, \mathrm{ddd}, J=11.8,7.4,4.6 \mathrm{~Hz}, \mathrm{NHCH})$, $3.86\left(3 \mathrm{H}, \mathrm{s}, \mathrm{OCH}_{3}\right), 3.52\left(1 \mathrm{H}, \mathrm{dd}, J=13.7,4.6 \mathrm{~Hz}, \mathrm{NHCHCH}_{2}\right), 3.24(1 \mathrm{H}, \mathrm{dd}, J=13.7,11.5 \mathrm{~Hz}, \mathrm{NHCHCH} 2)$.

${ }^{13} \mathrm{C}$ NMR (125.6 MHz, CDCl 3 ) $\delta 199.2(\mathrm{C}=0), 159.8(\mathrm{C}), 151.1(\mathrm{C}), 138.6(\mathrm{C}), 137.6(\mathrm{C}), 136.6(\mathrm{C}), 131.8(\mathrm{CH}), 131.2(\mathrm{CH}), 130.7(\mathrm{CH}), 129.44(\mathrm{CH}), 129.41$ $(\mathrm{CH}), 126.4(\mathrm{CH}), 126.3(\mathrm{CH}), 125.1(\mathrm{CH}), 124.0(\mathrm{CH}), 122.5(\mathrm{C}), 120.6(\mathrm{CH}), 119.1(\mathrm{CH}), 114.5(\mathrm{CH}), 58.7(\mathrm{CH}), 55.5\left(\mathrm{CH}_{3}\right), 37.7(\mathrm{CH})$.

HRMS (ESI) Exact mass calculated for $\mathrm{C}_{22} \mathrm{H}_{20} \mathrm{NO}_{5} \mathrm{~S}[\mathrm{M}+\mathrm{H}]^{+}: 410.1063$, found: 410.1057 .

The enantiomeric excess was determined by UPC 2 analysis on a Waters Amy1 column, 100:0 $\mathrm{CO}_{2} / i$-PrOH to $60: 40 \mathrm{CO}_{2} / i$-PrOH for 9 minutes with curve of "6", $\lambda=285 \mathrm{~nm}: \tau_{\text {minor }}=6.37 \mathrm{~min}, \tau_{\text {major }}=6.82 \min (73 \%$ ee $) .[\alpha]_{\mathrm{D}}{ }^{28}=+13.5\left(\mathrm{c}=0.8, \mathrm{CHCl}_{3}, 73 \%\right.$ ee $)$.

\section{(R)-(2-((2,2-dioxido-3,4-dihydrobenzo[e][1,2,3]oxathiazin-4-yl)methyl)phenyl)(4-tert-butylphenyl)methanone (3c)}

The title compound $\mathbf{3 c}$ was prepared according to the general procedure using $2 \mathbf{a}(9.2 \mathrm{mg}, 50 \mu \mathrm{mol})$ and (4-tert-butylphenyl)(o-tolyl)methanone 1c (25.2 $\mathrm{mg}, 100 \mu \mathrm{mol})$. The crude product was purified by FC (95:5 hexane/EtOAc) to afford amine $3 \mathrm{c}\left(16 \mathrm{mg}\right.$, 74\%, average of two runs) as a white solid. $\mathrm{R}_{\mathrm{f}}=0.36$ (9:1 hexane/EtOAc); $\mathrm{mp}=70-72{ }^{\circ} \mathrm{C}$ (hexane).

IR (ATR): 2919,1634 (C=0), 1599, 1487, 1456, 1370, 1187, 1106, 937, $751 \mathrm{~cm}^{-1}$.

1H NMR (500 MHz, CDCl $)$ ) 7.78-7.73 (2H, m, ArH), 7.61-7.55 (2H, m, ArH), 7.51-7.47 (2H, m, ArH), 7.47-7.43 (1H, m, ArH), 7.41-7.35 (2H, m, ArH), 7.32 $(1 \mathrm{H}, \mathrm{dddd}, J=8.2,7.3,1.6,0.7 \mathrm{~Hz}, \mathrm{ArH}), 7.24(1 \mathrm{H}, \mathrm{d}, J=7.6 \mathrm{~Hz}, \mathrm{NH}), 7.19(1 \mathrm{H}, \mathrm{td}, J=7.6,1.3 \mathrm{~Hz}, \operatorname{ArH}), 7.06(1 \mathrm{H}, \mathrm{dd}, J=8.2,1.3 \mathrm{~Hz}, \operatorname{ArH}), 5.14(1 \mathrm{H}, \mathrm{ddd}, J=11.9$, 7.6, $4.6 \mathrm{~Hz}, \mathrm{NHCH}), 3.51\left(1 \mathrm{H}, \mathrm{dd}, J=13.7,4.6 \mathrm{~Hz}, \mathrm{NHCHCH}_{2}\right), 3.21(1 \mathrm{H}, \mathrm{dd}, J=13.7,11.5 \mathrm{~Hz}, \mathrm{NHCHCH} 2), 1.36\left(9 \mathrm{H}, \mathrm{s}, \mathrm{C}\left(\mathrm{CH}_{3}\right)_{3}\right)$.

${ }^{13}$ C NMR (125.6 MHz, CDCl 3 ) $\delta 199.0$ (C=0), $157.9(\mathrm{C}), 151.2(\mathrm{C}), 137.9$ (C), $136.5(\mathrm{C}), 134.6(\mathrm{C}), 131.6(\mathrm{CH}), 131.1(\mathrm{CH}), 131.0(2 \times \mathrm{CH}), 130.5(\mathrm{CH}), 129.4$ $(\mathrm{CH}), 126.4(\mathrm{CH}), 126.3(\mathrm{CH}), 125.5(2 \times \mathrm{CH}), 125.1(\mathrm{CH}), 122.5(\mathrm{C}), 119.1(\mathrm{CH}), 58.6(\mathrm{CH}), 37.7\left(\mathrm{CH}_{2}\right), 35.3(\mathrm{C}), 31.0\left(3 \times \mathrm{CH}_{3}\right)$.

HRMS (ESI) Exact mass calculated for $\mathrm{C}_{25} \mathrm{H}_{25} \mathrm{NO}_{4} \mathrm{SNa}[\mathrm{M}+\mathrm{Na}]^{+}: 458.1398$, found: 458.1396. 
The enantiomeric excess was determined by UPC 2 analysis on a Waters Amy1 column, 100:0 $\mathrm{CO}_{2} / \mathrm{EtOH}$ to $60: 40 \mathrm{CO}_{2} / \mathrm{EtOH}$ for 9 minutes with curve of "6", $\lambda=261 \mathrm{~nm}: \tau_{\text {minor }}=5.90 \mathrm{~min}, \tau_{\text {major }}=6.07 \mathrm{~min}(77 \% \mathrm{ee}) \cdot[\alpha]_{\mathrm{D}}{ }^{28}=+30.0\left(\mathrm{c}=0.5, \mathrm{CHCl}_{3}, 77 \%\right.$ ee $)$.

\section{(R)-(2-((2,2-dioxido-3,4-dihydrobenzo[e][1,2,3]oxathiazin-4-yl)methyl)phenyl)(4-bromophenyl)methanone (3d)}

The title compound 3d was prepared according to the general procedure using $\mathbf{2 a}(9.2 \mathrm{mg}, 50 \mu \mathrm{mol})$ and (4-bromophenyl)(o-tolyl)methanone $\mathbf{1 d}(27.5 \mathrm{mg}$, $100 \mu \mathrm{mol})$. The crude product was purified by FC (95:5 hexane/EtOAc) to afford amine $\mathbf{3 d}\left(18 \mathrm{mg}, 79 \%\right.$, average of two runs) as a white solid. $\mathrm{R}_{\mathrm{f}}=0.30$ ( $9: 1$ hexane/EtOAc); $\mathrm{mp}=88-90{ }^{\circ} \mathrm{C}$ (hexane).

IR (ATR): 2922, 1645 (C=0), 1578, 1453, 1423, 1369, 1262, 1183, 1166, $750 \mathrm{~cm}^{-1}$.

1H NMR (500 MHz, CDCl $)$ $\delta$ 7.69-7.66 (2H, m, ArH), 7.65-7.58 (4H, m, ArH), 7.43-7.37 (3H, m, ArH), 7.33 (1H, dddd, J= 8.1, 7.3, 1.6, 0.7 Hz, ArH), 7.21 (1H, $\mathrm{td}, J=7.6,1.3 \mathrm{~Hz}, \mathrm{ArH}), 7.06(1 \mathrm{H}, \mathrm{dd}, J=8.3,1.2 \mathrm{~Hz}, \mathrm{ArH}), 6.92(1 \mathrm{H}, \mathrm{d}, J=7.7 \mathrm{~Hz}, \mathrm{NH}), 5.15(1 \mathrm{H}, \mathrm{ddd}, J=11.9,7.7,4.5 \mathrm{~Hz}, \mathrm{NHCH}), 3.52(1 \mathrm{H}, \mathrm{dd}, J=13.7,4.5 \mathrm{~Hz}$, $\left.\mathrm{CH}_{2}\right), 3.24\left(1 \mathrm{H}, \mathrm{dd}, J=13.7,11.5 \mathrm{~Hz}, \mathrm{CH}_{2}\right)$.

${ }^{13}$ C NMR (125.6 MHz, $\mathbf{C D C l}_{3}$ ) $\delta 198.3$ (C=0), 151.2 (C), $137.1(\mathrm{C}), 136.9(\mathrm{C}), 136.1(\mathrm{C}), 133.3(2 \times \mathrm{CH}), 132.1(\mathrm{CH}), 131.9(2 \times \mathrm{CH}), 131.5(\mathrm{CH}), 130.5(\mathrm{CH})$, $129.5(\mathrm{CH}), 129.3(\mathrm{C}), 126.6(\mathrm{CH}), 126.4(\mathrm{CH}), 125.2(\mathrm{CH}), 122.4(\mathrm{C}), 119.1(\mathrm{CH}), 58.7(\mathrm{CH}), 37.7\left(\mathrm{CH}_{2}\right)$.

HRMS (ESI) Exact mass calculated for $\mathrm{C}_{21} \mathrm{H}_{17} \mathrm{BrNO}_{4} \mathrm{~S}[\mathrm{M}+\mathrm{H}]^{+}: 458.0064$, found: 458.0056 .

The enantiomeric excess was determined by UPC 2 analysis on a Waters Amy1 column, 100:0 $\mathrm{CO}_{2} / \mathrm{EtOH}$ to $60: 40 \mathrm{CO}_{2} / \mathrm{EtOH}$ for 9 minutes with curve of "6", $\lambda=262 \mathrm{~nm}: \tau_{\text {major }}=6.44 \mathrm{~min}, \tau_{\text {minor }}=6.84 \mathrm{~min}(77 \% \mathrm{ee}) \cdot[\alpha]_{\mathrm{D}}{ }^{28}=+10.5\left(\mathrm{c}=1.0, \mathrm{CHCl}_{3}, 77 \%\right.$ ee $)$.

Recrystallation from 7:3 hexane/iso-propanol gave racemic crystals while the enantioenriched compound $\mathbf{3 d}$ (96\% ee) was recovered from the mother liquor.

\section{(R)-(2-((2,2-dioxido-3,4-dihydrobenzo[e][1,2,3]oxathiazin-4-yl)methyl)phenyl)(4-fluorophenyl)methanone (3e)}

The title compound 3e was prepared according to the general procedure using $\mathbf{2 a}(9.2 \mathrm{mg}, 50 \mu \mathrm{mol})$ and (4-fluorophenyl)(o-tolyl)methanone 1e (21 mg, $100 \mu \mathrm{mol})$. The crude product was purified by FC (95:5 hexane/EtOAc) to afford amine $3 \mathbf{e}\left(15.5 \mathrm{mg}\right.$, 82\%, average of two runs) as a white solid. $\mathrm{R}_{\mathrm{f}}=0.31$ (9:1 hexane/EtOAc); $\mathrm{mp}=119-121^{\circ} \mathrm{C}$ (hexane).

IR (ATR): 2922, 1643 (C=0), 1593, 1486, 1455, 1424, 1363, 1183, 1110, $751 \mathrm{~cm}^{-1}$.

${ }^{1} \mathbf{H}$ NMR (500 MHz, $\left.\mathbf{C D C l}_{3}\right) \delta 7.85(2 \mathrm{H}, \mathrm{dd}, J=8.9,5.4 \mathrm{~Hz}, \mathrm{ArH}), 7.59$ (2H, m, ArH), 7.42-7.37 (3H, m, ArH), 7.33 (1H, dddd, $J=8.2,7.4,1.7,0.7$ Hz, ArH), 7.23$7.13(3 \mathrm{H}, \mathrm{m}, \mathrm{ArH}), 7.09-7.02(2 \mathrm{H}, \mathrm{m}, \mathrm{ArH}), 5.15(1 \mathrm{H}, \mathrm{ddd}, J=11.9,7.7,4.5 \mathrm{~Hz}, \mathrm{CHNH}), 3.52(1 \mathrm{H}, \mathrm{dd}, J=13.7,4.6 \mathrm{~Hz}, \mathrm{CH} 2 \mathrm{CHNH}), 3.23(1 \mathrm{H}, \mathrm{dd}, J=13.7,11.5$ $\mathrm{Hz}, \mathrm{CH}_{2} \mathrm{CHNH}$ )

${ }^{13} \mathrm{C}$ NMR (125.6 MHz, CDCl 3 ) $\delta 197.8$ (C=0), 166.2 (d, $\left.J=257 \mathrm{~Hz}, \mathrm{C}\right), 151.2(\mathrm{C}), 137.3(\mathrm{C}), 136.7$ (C), 133.7 (CH), 133.6 (d, J = 2.9 Hz, C), 133.5 (CH), 131.9 $(\mathrm{CH}), 131.3(\mathrm{CH}), 130.4(\mathrm{CH}), 129.5(\mathrm{CH}), 126.4(\mathrm{~d}, J=26.2 \mathrm{~Hz}, 2 \times \mathrm{CH}), 125.1(\mathrm{CH}), 122.4(\mathrm{CH}), 119.1(\mathrm{CH}), 115.8(\mathrm{~d}, J=22.1 \mathrm{~Hz}, 2 \times \mathrm{CH}), 58.7(\mathrm{CH}), 37.7(\mathrm{CH} 2)$. ${ }^{19}$ F NMR (376 MHz, $\left.\mathrm{CDCl}_{3}\right) \delta-103.4(\mathrm{tt}, J=8.3,5.4 \mathrm{~Hz})$.

HRMS (ESI) Exact mass calculated for $\mathrm{C}_{21} \mathrm{H}_{17} \mathrm{FNO}_{4} \mathrm{~S}[\mathrm{M}+\mathrm{H}]^{+}: 398.0857$, found: 398.0854 .

The enantiomeric excess was determined by UPC 2 analysis on a Waters Cel2 column, 100:0 $\mathrm{CO}_{2} / \mathrm{MeCN}$ to $60: 40 \mathrm{CO}_{2} / \mathrm{MeCN}$ for 9 minutes with curve of "6", $\lambda=254 \mathrm{~nm}: \tau_{\text {minor }}=4.63 \mathrm{~min}, \tau_{\text {major }}=4.75 \mathrm{~min}(75 \%$ ee $) \cdot[\alpha]_{\mathrm{D}}{ }^{28}=+16.2\left(\mathrm{c}=1.0, \mathrm{CHCl}_{3}, 75 \%\right.$ ee $)$.

\section{(R)-(2-((2,2-dioxido-3,4-dihydrobenzo[e][1,2,3]oxathiazin-4-yl)methyl)phenyl)(3-methoxyphenyl)methanone (3f)}

The title compound $\mathbf{3 f}$ was prepared according to the general procedure using $\mathbf{2 a}(9.2 \mathrm{mg}, 50 \mu \mathrm{mol})$ and (3-methoxyphenyl)( $o$-tolyl)methanone $\mathbf{1 f}(22.6 \mathrm{mg}$, $100 \mu \mathrm{mol})$. The crude product was purified by FC (95:5 hexane/EtOAc) to afford amine $\mathbf{3 f}\left(15 \mathrm{mg}\right.$, $73 \%$, average of two runs) as a white solid. $\mathrm{R}_{\mathrm{f}}=0.22(9: 1$ hexane/EtOAc); $m p=53-55^{\circ} \mathrm{C}$ (hexane).

IR (ATR): 2922, 1635 (C=0), 1576, 1483, 1454, 1373, 1269, 1183, 1166, $751 \mathrm{~cm}^{-1}$

${ }^{1} \mathbf{H}$ NMR (500 MHz, CDCl 3$) \delta$ 7.63-7.58 (2H, m, ArH), 7.47-7.43 (1H, m, ArH), 7.43-7.34 (4H, m, ArH), 7.33 (1H, dddd, J= 8.2,7.3, 1.7, 0.7 Hz, ArH). 7.29 (1H, $\mathrm{dt}, J=7.7,1.3 \mathrm{~Hz}, \mathrm{ArH}), 7.24-7.15(2 \mathrm{H}, \mathrm{m}, \mathrm{ArH}), 7.12,(1 \mathrm{H}, \mathrm{d}, J=7.6 \mathrm{~Hz}, \mathrm{NH}), 7.06(1 \mathrm{H}, \mathrm{dd}, J=8.3,1.2 \mathrm{~Hz}, \mathrm{ArH}), 5.15(1 \mathrm{H}, \mathrm{ddd}, J=11.9,7.6,4.6 \mathrm{~Hz}, \mathrm{NHCH}), 3.86$ $\left(3 \mathrm{H}, \mathrm{s}, \mathrm{OCH}_{3}\right), 3.52\left(1 \mathrm{H}, \mathrm{dd}, J=13.7,4.6 \mathrm{~Hz}, \mathrm{NHCHCH}_{2}\right), 3.24\left(1 \mathrm{H}, \mathrm{dd}, J=13.7,11.6 \mathrm{~Hz}, \mathrm{NHCHCH}_{2}\right)$.

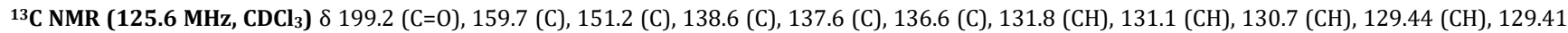
$(\mathrm{CH}), 126.4(\mathrm{CH}), 126.3(\mathrm{CH}), 125.1(\mathrm{CH}), 124.0(\mathrm{CH}), 122.4(\mathrm{C}), 120.6(\mathrm{CH}), 119.1(\mathrm{CH}), 114.5(\mathrm{CH}), 58.7\left(\mathrm{CH}^{2}, 55.5\left(\mathrm{CH}_{3}\right), 37.7\left(\mathrm{CH}_{2}\right)\right.$.

HRMS (ESI) Exact mass calculated for $\mathrm{C}_{22} \mathrm{H}_{19} \mathrm{NO}_{5} \mathrm{SNa}[\mathrm{M}+\mathrm{Na}]^{+}: 432.0876$, found: 432.0878.

The enantiomeric excess was determined by UPC 2 analysis on a Waters Amy1 column, 100:0 $\mathrm{CO}_{2} / i$-PrOH to $60: 40 \mathrm{CO}_{2} / i$-PrOH for 9 minutes with curve of "6", $\lambda=257 \mathrm{~nm}: \tau_{\text {minor }}=5.87 \mathrm{~min}, \tau_{\text {major }}=6.06 \mathrm{~min}(69 \%$ ee $) .[\alpha]_{\mathrm{D}}^{28}=+27.34\left(\mathrm{c}=0.5, \mathrm{CHCl}_{3}, 69 \%\right.$ ee $)$.

\section{(R)-(2-((2,2-dioxido-3,4-dihydrobenzo[e][1,2,3]oxathiazin-4-yl)methyl)phenyl)(3-chlorophenyl)methanone (3g)}

The title compound $\mathbf{3 g}$ was prepared according to the general procedure using $\mathbf{2 a}(9.2 \mathrm{mg}, 50 \mu \mathrm{mol})$ and (3-chlorophenyl)( $o$-tolyl)methanone $\mathbf{1 g}$ (23 mg, $100 \mu \mathrm{mol})$. The crude product was purified by FC (95:5 hexane/EtOAc) to afford amine $\mathbf{3 g}\left(14.5 \mathrm{mg}, 70 \%\right.$, average of two runs) as a white solid. $\mathrm{R}_{\mathrm{f}}=0.33$ (9:1 hexane/EtOAc); $\mathrm{mp}=39-41^{\circ} \mathrm{C}$ (hexane).

IR (ATR): 2922, 1645 (C=0), 1567, 1484, 1452, 1419, 1372, 1257, 1184, $747 \mathrm{~cm}^{-1}$.

${ }^{1} \mathbf{H}$ NMR (500 MHz, CDCl 3 ) $\delta 7.79(1 \mathrm{H}, \mathrm{t}, J=1.9 \mathrm{~Hz}, \mathrm{ArH}), 7.68(1 \mathrm{H}, \mathrm{dt}, J=7.8,1.3 \mathrm{~Hz}, \operatorname{ArH}), 7.64-7.58(3 \mathrm{H}, \mathrm{m}, \operatorname{ArH}), 7.48-7.37(4 \mathrm{H}, \mathrm{m}, \operatorname{ArH}), 7.33(1 \mathrm{H}, \mathrm{dddd}, J$ $=8.1,7.3,1.6,0.7 \mathrm{~Hz}, \operatorname{ArH}), 7.21(1 \mathrm{H}, \mathrm{td}, J=7.6,1.3 \mathrm{~Hz}, \operatorname{ArH}), 7.06(1 \mathrm{H}, \mathrm{dd}, J=8.3,1.2 \mathrm{~Hz}, \operatorname{ArH}), 6.77(1 \mathrm{H}, \mathrm{d}, J=7.7 \mathrm{~Hz}, \mathrm{NH}), 5.26(1 \mathrm{H}, \mathrm{ddd}, J=11.9,7.7,4.5 \mathrm{~Hz}$, $\mathrm{NHCH}), 3.52\left(1 \mathrm{H}, \mathrm{dd}, J=13.7,4.5 \mathrm{~Hz}, \mathrm{NHCHCH}_{2}\right), 3.26\left(1 \mathrm{H}, \mathrm{dd}, J=13.7,11.5 \mathrm{~Hz}, \mathrm{NHCHCH}_{2}\right)$. 
${ }^{13}$ C NMR (125.6 MHz, CDCl$\left._{3}\right) \delta 198.0$ (C=0), $151.2(\mathrm{C}), 139.0(\mathrm{C}), 137.0(\mathrm{C}), 136.9(\mathrm{C}), 134.9(\mathrm{C}), 133.7(\mathrm{CH}), 132.2(\mathrm{CH}), 131.6(\mathrm{CH}), 130.7(\mathrm{CH}), 130.6(\mathrm{CH})$, $129.9(\mathrm{CH}), 129.5(\mathrm{CH}), 128.9(\mathrm{CH}), 126.6(\mathrm{CH}), 126.4(\mathrm{CH}), 125.2(\mathrm{CH}), 122.3(\mathrm{C}), 119.1(\mathrm{CH}), 58.7(\mathrm{CH}), 37.7\left(\mathrm{CH}_{2}\right)$.

HRMS (ESI) Exact mass calculated for $\mathrm{C}_{21} \mathrm{H}_{16} \mathrm{ClNO}_{4} \mathrm{SNa}[\mathrm{M}+\mathrm{Na}]^{+}: 436.0381$, found: 436.0384 .

The enantiomeric excess was determined by UPC 2 analysis on a Waters Amy1 column, 100:0 $\mathrm{CO}_{2} / \mathrm{EtOH}$ to $60: 40 \mathrm{CO}_{2} / \mathrm{EtOH}_{\mathrm{H}}$ for 9 minutes with curve of "6", $\lambda=254 \mathrm{~nm}: \tau_{\text {minor }}=5.79 \mathrm{~min}, \tau_{\text {major }}=5.99 \mathrm{~min}(72 \% \mathrm{ee}) .[\alpha]_{\mathrm{D}}{ }^{28}=+17.3\left(\mathrm{c}=0.7, \mathrm{CHCl}_{3}, 72 \%\right.$ ee $)$.

\section{(R)-(2-((2,2-dioxido-3,4-dihydrobenzo[e][1,2,3]oxathiazin-4-yl)methyl)phenyl)(3-bromophenyl)methanone (3h)}

The title compound $\mathbf{3 h}$ was prepared according to the general procedure using $\mathbf{2 a}$ ( $9.2 \mathrm{mg}, 50 \mu \mathrm{mol})$ and (3-bromophenyl)(o-tolyl)methanone $\mathbf{1 h}$ (27.5 mg, $100 \mu \mathrm{mol})$. The crude product was purified by FC (95:5 hexane/EtOAc) to afford amine $\mathbf{3 h}\left(17 \mathrm{mg}, 74 \%\right.$, average of two runs) as a white solid. $\mathrm{R}_{\mathrm{f}}=0.34(9: 1$ hexane/EtOAc); $\mathrm{mp}=60-62^{\circ} \mathrm{C}$ (hexane).

IR (ATR): 2921, 1645 (C=0), 1558, 1485, 1449, 1418, 1373, 1256, 1185, $739 \mathrm{~cm}^{-1}$.

1H NMR (500 MHz, $\left.\mathbf{C D C l}_{3}\right) \delta 7.94(1 \mathrm{H}, \mathrm{t} . J=1.8 \mathrm{~Hz}, \mathrm{ArH}), 7.76(1 \mathrm{H}, \mathrm{ddd}, J=8.0,2.0,1.1 \mathrm{~Hz}, \operatorname{ArH}), 7.72(1 \mathrm{H}, \mathrm{ddd}, J=7.8,1.7,1.1 \mathrm{~Hz}, \mathrm{ArH}), 7.65-7.59(2 \mathrm{H}, \mathrm{m}$, ArH), 7.45-7.30 (5H, m, ArH), 7.21 (1H, td, $J=7.6,1.3 \mathrm{~Hz}, \mathrm{ArH}), 7.06(1 \mathrm{H}, \mathrm{dd}, J=8.3,1.2 \mathrm{~Hz}, \mathrm{ArH}), 6.76(1 \mathrm{H}, \mathrm{d}, J=7.7 \mathrm{~Hz}, \mathrm{NH}), 5.16(1 \mathrm{H}, \mathrm{ddd}, J=11.9,7.7,4.5$ $\mathrm{Hz}, \mathrm{NHCH}), 3.52\left(1 \mathrm{H}, \mathrm{dd}, J=13.7,4.5 \mathrm{~Hz}, \mathrm{NHCHCH}_{2}\right), 3.26\left(1 \mathrm{H}, \mathrm{dd}, J=13.7,11.5 \mathrm{~Hz} \mathrm{NHCHCH}_{2}\right.$ ).

${ }^{13}$ C NMR (125.6 MHz, CDCl $)$ ) $\delta 197.9$ (C=0), 151.2 (C), 139.2 (C), $137.0(\mathrm{C}), 136.9(\mathrm{C}), 136.6(\mathrm{CH}), 133.6(\mathrm{CH}), 132.2(\mathrm{CH}), 131.6(\mathrm{CH}), 130.6(\mathrm{CH}), 130.1$ $(\mathrm{CH}), 129.5(\mathrm{CH}), 129.4(\mathrm{CH}), 126.7(\mathrm{CH}), 126.4(\mathrm{CH}), 125.2(\mathrm{CH}), 122.8(\mathrm{C}), 122.3(\mathrm{C}), 119.1(\mathrm{CH}), 58.7(\mathrm{CH}), 37.7\left(\mathrm{CH}_{2}\right)$.

HRMS (ESI) Exact mass calculated for $\mathrm{C}_{21} \mathrm{H}_{16} \mathrm{BrNO}_{4} \mathrm{SNa}[\mathrm{M}+\mathrm{Na}]^{+}: 479.9876$, found: 479.9861.

The enantiomeric excess was determined by UPC 2 analysis on a Waters Amy1 column, 100:0 $\mathrm{CO}_{2} / \mathrm{EtOH}$ to $60: 40 \mathrm{CO}_{2} / \mathrm{EtOH}_{\text {for }} 9$ minutes with curve of "6", $\lambda=254 \mathrm{~nm}: \tau_{\text {major }}=6.07 \mathrm{~min}, \tau_{\text {minor }}=6.30 \mathrm{~min}(71 \%$ ee $) \cdot[\alpha]_{\mathrm{D}}{ }^{28}=+7.9\left(\mathrm{c}=1.0, \mathrm{CHCl}_{3}, 71 \%\right.$ ee $)$.

\section{(R)-(2-((2,2-dioxido-3,4-dihydrobenzo[e][1,2,3]oxathiazin-4-yl)methyl)phenyl)(2,3-dimethoxyphenyl)methanone (3i)}

The title compound $\mathbf{3 i}$ was prepared according to the general procedure using $\mathbf{2 a}(9.2 \mathrm{mg}, 50 \mu \mathrm{mol})$ and (2,3.dimethoxyphenyl)(o-tolyl)methanone $\mathbf{1 i}$ (25.6 $\mathrm{mg}, 100 \mu \mathrm{mol})$. The crude product was purified by FC ( $95: 5$ hexane/EtOAc) to afford amine $3 \mathbf{i}\left(17 \mathrm{mg}\right.$, $77 \%$, average of two runs) as a white solid. $\mathrm{R}_{\mathrm{f}}=0.19$ (9:1 hexane/EtOAc); $\mathrm{mp}=45-47^{\circ} \mathrm{C}$ (hexane).

IR (ATR): 2924, 1652 (C=0), 1576, 1473, 1423, 1373, 1311, 1263, 1165, $750 \mathrm{~cm}^{-1}$.

${ }^{1} \mathbf{H}$ NMR (500 MHz, $\mathbf{C D C l}_{3}$ ) $\delta$ 7.58-7.46 (3H, m, ArH), 7.38-7.29 (3H, m, ArH), 7.22 (1H, td, J= 7.6, 1.3 Hz, ArH), 7.15 (1H, dd, J= 8.4, 7.2 Hz, ArH), 7.12-7.07 $(2 \mathrm{H}, \mathrm{m}, \mathrm{ArH}), 7.05(1 \mathrm{H}, \mathrm{dd}, J=8.2,1.2 \mathrm{~Hz}, \mathrm{ArH}), 6.40(1 \mathrm{H}, \mathrm{d}, J=7.5 \mathrm{~Hz}, \mathrm{NH}), 5.19(1 \mathrm{H}, \mathrm{ddd}, J=11.7,7.6,4.3 \mathrm{~Hz}, \mathrm{NHCH}), 3.88(3 \mathrm{H}, \mathrm{s}, \mathrm{OCH} 3), 3.73(1 \mathrm{H}, \mathrm{dd}, J=$ $\left.13.7,4.3 \mathrm{~Hz}, \mathrm{NHCHCH}_{2}\right), 3.48\left(3 \mathrm{H}, \mathrm{s}, \mathrm{OCH}_{3}\right), 3.43\left(1 \mathrm{H}, \mathrm{dd}, J=13.7,11.6 \mathrm{~Hz}, \mathrm{NHCHCH}_{2}\right)$.

${ }^{13} \mathrm{C}$ NMR (125.6 MHz, $\mathbf{C D C l}_{3}$ ) $\delta 200.4(\mathrm{C}=0), 152.9(\mathrm{C}), 151.2(\mathrm{C}), 147.9(\mathrm{C}), 139.5(\mathrm{C}), 136.3(\mathrm{C}), 134.2(\mathrm{C}), 131.9(\mathrm{CH}), 131.8(\mathrm{CH}), 130.7(\mathrm{CH}), 129.4(\mathrm{CH})$, $126.8(\mathrm{CH}), 126.6(\mathrm{CH}), 125.2(\mathrm{CH}), 124.0(\mathrm{CH}), 122.5(\mathrm{C}), 121.4(\mathrm{CH}), 119.0(\mathrm{CH}), 116.0(\mathrm{CH}), 61.0\left(\mathrm{CH}_{3}\right), 58.7\left(\mathrm{CH}^{2}, 56.0\left(\mathrm{CH}_{3}\right), 38.0(\mathrm{CH})\right.$.

HRMS (ESI) Exact mass calculated for $\mathrm{C}_{23} \mathrm{H}_{21} \mathrm{NO}_{6} \mathrm{SNa}[\mathrm{M}+\mathrm{Na}]^{+}: 462.0982$, found: 462.0983.

The enantiomeric excess was determined by UPC 2 analysis on a Waters Amy1 column, 100:0 $\mathrm{CO}_{2} / \mathrm{EtOH}$ to $60: 40 \mathrm{CO} / \mathrm{EtOH}_{2}$ for 9 minutes with curve of "6", $\lambda=254 \mathrm{~nm}: \tau_{\text {major }}=5.70 \mathrm{~min}, \tau_{\text {minor }}=5.88 \mathrm{~min}(39 \% \mathrm{ee}) .[\alpha]_{\mathrm{D}}^{28}=+22.1\left(\mathrm{c}=0.7, \mathrm{CHCl}_{3}, 39 \%\right.$ ee $)$.

\section{$(R)-(2-((2,2-d i o x i d o-3,4-d i h y d r o b e n z o[e][1,2,3]$ oxathiazin-4-yl)ethyl)phenyl)(phenyl)methanone (3j)}

The title compound $\mathbf{3} \mathbf{j}$ was prepared according to the general procedure using $\mathbf{2 a}(9.2 \mathrm{mg}, 50 \mu \mathrm{mol})$ and (2-ethyl)(phenyl)methanone $\mathbf{1 j}(21.0 \mathrm{mg}, 100 \mu \mathrm{mol})$. The crude product was purified by FC ( $95: 5$ hexane/EtOAc) to afford amine $3 \mathbf{j}$ (13 mg, 66\%, average of two runs) as an inseparable mixture of diastereomers (1.3:1 d.r.) and as a white solid. $\mathrm{R}_{\mathrm{f}}=0.30$ (9:1 hexane/EtOAc).

IR (ATR): 2922, 1652, (C=0), 1448, 1378, 1314, 1268, 1189, 1102, 927, $758 \mathrm{~cm}^{-1}$.

Major diastereoisomer: ${ }^{1} \mathbf{H}$ NMR (500 MHz, $\left.\mathbf{C D C l}_{3}\right) \delta$ 7.88-7.76 (2H, m, ArH), 7.68-7.56 (1H, m, ArH), 7.54-7.43 (3H, m, ArH), 7.41-7.29 (3H, m, ArH), 7.25$7.15(3 \mathrm{H}, \mathrm{m}, \mathrm{ArH}), 7.01(1 \mathrm{H}, \mathrm{dd}, J=8.2,1.2 \mathrm{~Hz}, \mathrm{ArH}), 5.54(1 \mathrm{H}, \mathrm{d}, J=6.5 \mathrm{~Hz}, \mathrm{NH}), 5.11(1 \mathrm{H}, \mathrm{dd}, J=6.4,4.3 \mathrm{~Hz}, \mathrm{NHCH}), 4.15-4.06(1 \mathrm{H}, \mathrm{m}, \mathrm{NHCHCH}), 1.28(3 \mathrm{H}$, $\left.\mathrm{d}, J=2.9 \mathrm{~Hz}, \mathrm{CH}_{3}\right)$.

${ }^{13} \mathrm{C}$ NMR (125.6 MHz, CDCl $) \delta 199.0$ (C=0), 151.6 (C), 140.7 (C), 137.9 (C),137.7 (C), 130.9 (CH), 130.5 (2 x CH), 129.7 (CH), 129.43 (CH), 129.2 (CH), 128.7 $(\mathrm{CH}), 128.6(2 \mathrm{x} \mathrm{CH}), 127.0(\mathrm{CH}), 126.5(\mathrm{CH}), 125.5(\mathrm{CH}), 121.9(\mathrm{C}), 119.1(\mathrm{CH}), 62.1(\mathrm{CH}), 38.8(\mathrm{CH}), 14.4\left(\mathrm{CH}_{3}\right)$.

Minor diastereoisomer: ${ }^{1} \mathbf{H}$ NMR (500 MHz, $\left.\mathbf{C D C l}_{3}\right) \delta$ 7.78-7.70 (2H, m, ArH), 7.65-7.58 (2H, m, ArH), 7.53-7.43 (3H, m, ArH), 7.40-7.29 (3H, m, ArH), 7.21 $(1 \mathrm{H}, \mathrm{tdd}, J=7.5,1.6,0.7 \mathrm{~Hz}, \mathrm{ArH}), 7.07-6.99(2 \mathrm{H}, \mathrm{m}, \mathrm{ArH}), 6.13(1 \mathrm{H}, \mathrm{d}, J=7.8 \mathrm{~Hz}, \mathrm{NH}), 4.91(1 \mathrm{H}, \mathrm{t}, J=7.8 \mathrm{~Hz}, \mathrm{NHCH}), 3.93(1 \mathrm{H}, \mathrm{app} \mathrm{p}, J=7.1 \mathrm{~Hz}, \mathrm{NHCHCH})$, $1.58\left(3 \mathrm{H}, \mathrm{d}, J=7.0 \mathrm{~Hz}, \mathrm{CH}_{3}\right)$,

$\left.{ }^{13} \mathrm{C} \mathrm{NMR} \mathrm{(125.6} \mathrm{MHz,} \mathrm{CDCl}\right) ~ \delta 199.2(\mathrm{C}=0), 151.7$ (C), 141.6 (C), 138.1 (C), 137.0 (C), 133.7 (CH), 131.5 (CH), 130.8 (2 x CH), 129.8 (CH), 129.42 (CH), 128.4 $(2 \times \mathrm{CH}), 128.2(\mathrm{CH}), 127.5(\mathrm{CH}), 126.3(\mathrm{CH}), 124.9(\mathrm{CH}), 122.6(\mathrm{C}), 119.2(\mathrm{CH}), 62.2(\mathrm{CH}), 39.0(\mathrm{CH}), 20.5\left(\mathrm{CH}_{3}\right)$.

HRMS (ESI) Exact mass calculated for $\mathrm{C}_{22} \mathrm{H}_{19} \mathrm{NO}_{4} \mathrm{SNa}$ [M+Na] $]^{+}: 416.0927$, found: 416.0926.

The enantiomeric excess of the major diastereoisomer was determined by UPC 2 analysis on a Waters Amy1 column, 100:0 $\mathrm{CO}_{2} / \mathrm{MeOH}$ to $60: 40 \mathrm{CO} / \mathrm{MeOH}$ for 9 minutes with curve of " 6 ", $\lambda=249 \mathrm{~nm}$ : $\tau_{\text {major }}=5.62 \mathrm{~min}, \tau_{\operatorname{minor}}=6.20 \mathrm{~min}(68 \%$ ee$)$. The enantiomeric excess of the minor diastereoisomer was determined by UPC ${ }^{2}$ analysis on a Waters Amy1 column, 100:0 $\mathrm{CO}_{2} / \mathrm{MeOH}$ to $60: 40 \mathrm{CO}_{2} / \mathrm{MeOH}$ for 9 minutes with curve of “6”, $\lambda=250 \mathrm{~nm}: \tau_{\text {minor }}=5.64 \mathrm{~min}$, $\tau_{\text {major }}=6.08 \mathrm{~min}(60 \%$ ee $) \cdot[\alpha]_{\mathrm{D}}^{28}=+15.4\left(\mathrm{c}=0.8, \mathrm{CHCl}_{3}, 1.3: 1 \mathrm{dr}, 68 \%\right.$ ee $)$.

(R)-(2-((2,2-dioxido-3,4-dihydrobenzo[e][1,2,3]oxathiazin-4-yl)methyl)-4-(trifluoromethyl)phenyl)(phenyl)methanone (3k) 
The title compound $\mathbf{3 k}$ was prepared according to the general procedure using $\mathbf{2 a}$ (9.2 mg, 50 $\mu$ mol) and (2-methyl-4(trifluoromethyl)phenyl)(phenyl)methanone 1k $(26.4 \mathrm{mg}, 100 \mu \mathrm{mol})$. The crude product was purified by FC ( $95: 5$ hexane/EtOAc) to afford amine $3 \mathbf{k}$ (18.8 $\mathrm{mg}, 84 \%$, average of two runs) as a white solid. $\mathrm{R}_{\mathrm{f}}=0.31$ (9:1 hexane/EtOAc); $\mathrm{mp}=167-169^{\circ} \mathrm{C}$ (hexane).

IR (ATR): 3252, 2929, 1658 )C=0), 1484, 1424, 1374, 1164,1121, 1075, 1075, $756 \mathrm{~cm}^{-1}$.

1H NMR (500 MHz, CDCl $)$ ) 7.84- 7.77 (3H, m, ArH), 7.70-7.63 (2H, m, ArH), 7.57-7.48 (3H, m, ArH), 7.36-7.30 (2H, m, ArH), 7.23-7.18 (1H, m ArH), 7.08$7.02(1 \mathrm{H}, \mathrm{m}, \mathrm{ArH}), 6.69(1 \mathrm{H}, \mathrm{d}, J=7.7 \mathrm{~Hz} \mathrm{NH}), 5.15(1 \mathrm{H}, \mathrm{ddd}, J=11.8,7.7,4.8 \mathrm{~Hz}, \mathrm{CHNH}), 3.53(1 \mathrm{H}, \mathrm{dd}, J=13.8,4.4 \mathrm{~Hz}, \mathrm{CH} 2 \mathrm{CHNH}), 3.30(1 \mathrm{H}, \mathrm{dd}, J=13.8,11.4$ $\left.\mathrm{Hz}, \mathrm{CH}_{2} \mathrm{CHNH}\right)$.

${ }^{13}$ C NMR (125.6 MHz, CDCl$_{3}$ ) $\delta 198.3$ (C=0), 151.1 (C), 140.9 (C), 137.4 (C), 136.5 (C), 134.5 (CH), 133.2 (q, J=33.1 Hz, C), 130.8 (2 x CH), 130.5 (CH), 129.7 $(\mathrm{CH}), 128.8(2 \times \mathrm{CH}), 128.2(\mathrm{q}, J=3.8 \mathrm{~Hz}, \mathrm{CH}), 126.4(\mathrm{CH}), 125.3(\mathrm{CH}), 123.5(\mathrm{q}, J=3.8 \mathrm{~Hz}, \mathrm{CH}), 123.4\left(\mathrm{q}, J=272.8 \mathrm{~Hz}, \mathrm{CF}_{3}\right), 121.9(\mathrm{C}), 119.2(\mathrm{CH}), 58.5(\mathrm{CH})$, $37.7\left(\mathrm{CH}_{2}\right)$.

${ }^{19}$ F NMR (376 MHz, $\left.\mathrm{CDCl}_{3}\right) \delta-63.0\left(\mathrm{~s}, \mathrm{CF}_{3}\right)$.

HRMS (ESI) Exact mass calculated for $\mathrm{C}_{22} \mathrm{H}_{16} \mathrm{~F}_{3} \mathrm{NNaO}_{4} \mathrm{~S}$ [M+Na] ${ }^{+}: 470.0644$, found: 470.0649.

The enantiomeric excess was determined by UPC 2 analysis on a Waters Amy1 column, 100:0 $\mathrm{CO}_{2} / i$-PrOH to $60: 40 \mathrm{CO}_{2} / i$-PrOH for 9 minutes with curve of "6", $\lambda=251 \mathrm{~nm}: \tau_{\text {minor }}=4.59 \mathrm{~min}, \tau_{\text {major }}=4.78 \mathrm{~min}(37 \%$ ee $) .[\alpha]_{\mathrm{D}}^{28}=+1.4\left(\mathrm{c}=0.93, \mathrm{CHCl}_{3}, 37 \%\right.$ ee $)$.

\section{(R)-(2-((6-methyl-2,2-dioxido-3,4-dihydrobenzo[e][1,2,3]oxathiazin-4-yl)methyl)phenyl)(phenyl)methanone (7a)}

The title compound $\mathbf{7 a}$ was prepared according to the general procedure using $\mathbf{2 b}(9.9 \mathrm{mg}, 50 \mu \mathrm{mol})$ and 2-methylbenzophenone $\mathbf{1 a}(18 \mu \mathrm{L}, 100 \mu \mathrm{mol})$. The crude product was purified by FC (95:5 hexane/EtOAc) to afford amine 7a (15.6 mg, 79\%, average of two runs) as a white solid. $\mathrm{R}_{\mathrm{f}}=0.30$ (9:1 hexane/EtOAc); $\mathrm{mp}=50-52{ }^{\circ} \mathrm{C}$ (hexane).

IR (ATR): 2921, 1651 (C=0), 1488, 1404, 1372, 1266, 1173, 1114, 847, $762 \mathrm{~cm}^{-1}$.

${ }^{1} \mathbf{H}$ NMR (500 MHz, CDCl $)$ $\delta$ 7.82- 7.77 (2H, m, ArH), 7.63 (1H, ddt, J = 8.7, 7.4, 1.3 Hz, ArH), 7.61-7.55 (1H, m, ArH), 7.50-7.46 (2H, m, ArH), 7.44-7.41 (1H, m ArH), 7.40-7.35 (1H, m, ArH), 7.15-7.13 (1H, m, ArH), 7.12-7.07 (1H, m, Hz, ArH), $6.99(1 \mathrm{H}, \mathrm{d}, J=7.6 \mathrm{NH}), 6.93(1 \mathrm{H}, \mathrm{d}, J=8.4 \mathrm{~Hz}, \mathrm{ArH}), 6.97(1 \mathrm{H}, \mathrm{d}, J=8.6$ $\mathrm{Hz}, \mathrm{ArH}), 5.09$ (1H, ddd, $J=12.0,7.6,4.6 \mathrm{~Hz}, \mathrm{CHNH}), 3.51\left(1 \mathrm{H}, \mathrm{dd}, J=13.7,4.6 \mathrm{~Hz}, \mathrm{CH}_{2} \mathrm{CHNH}\right), 3.22(1 \mathrm{H}, \mathrm{dd}, J=13.7,11.5 \mathrm{~Hz}, \mathrm{CH} 2 \mathrm{CHNH}), 2.32(3 \mathrm{H}, \mathrm{s}, \mathrm{CH})$.

${ }^{13} \mathrm{C}$ NMR (125.6 MHz, CDCl 3$) ~ \delta 199.4(\mathrm{C}=0), 149.1(\mathrm{C}), 137.6(\mathrm{C}), 137.3(\mathrm{C}), 136.7(\mathrm{C}), 134.8(\mathrm{C}), 133.8(\mathrm{CH}), 131.7(\mathrm{CH}), 131.2(\mathrm{CH}), 130.9(2 \times \mathrm{CH}), 130.6$ $(\mathrm{CH}), 130.0(\mathrm{CH}), 128.5(2 \times \mathrm{CH}), 126.6(\mathrm{CH}), 126.4(\mathrm{CH}), 122.0(\mathrm{C}), 118.8(\mathrm{CH}), 58.7(\mathrm{CH}), 37.7\left(\mathrm{CH}_{2}\right), 20.9\left(\mathrm{CH}_{3}\right)$.

HRMS (ESI) Exact mass calculated for $\mathrm{C}_{22} \mathrm{H}_{19} \mathrm{NNaO}_{4} \mathrm{~S}[\mathrm{M}+\mathrm{Na}]^{+}: 416.0927$, found: 416.0932 .

The enantiomeric excess was determined by UPC 2 analysis on a Waters Amy1 column, 100:0 $\mathrm{CO}_{2} / \mathrm{MeOH}$ to $60: 40 \mathrm{CO}_{2} / \mathrm{MeOH}_{\text {for }} 9$ minutes with curve of "6", $\lambda=251 \mathrm{~nm}: \tau_{\text {minor }}=5.46 \mathrm{~min}, \tau_{\text {major }}=5.69 \mathrm{~min}(64 \%$ ee $) .[\alpha]_{\mathrm{D}}^{28}=+7.2\left(\mathrm{c}=0.98, \mathrm{CHCl}_{3}, 64 \%\right.$ ee $)$.

\section{(R)-(2-((7-methyl-2,2-dioxido-3,4-dihydrobenzo[e][1,2,3]oxathiazin-4-yl)methyl)phenyl)(phenyl)methanone (7b)}

The title compound $\mathbf{7 b}$ was prepared according to the general procedure using $\mathbf{2 c}(9.9 \mathrm{mg}, 50 \mu \mathrm{mol})$ and 2 -methylbenzophenone $\mathbf{1 a}(18 \mu \mathrm{L}, 100 \mu \mathrm{mol})$. The crude product was purified by FC (95:5 hexane/EtOAc) to afford amine $\mathbf{7 b}\left(15.6 \mathrm{mg}, 79 \%\right.$, average of two runs) as a white solid. $\mathrm{R}_{\mathrm{f}}=0.32$ (9:1 hexane/EtOAc); $\mathrm{mp}=54-56^{\circ} \mathrm{C}$ (hexane).

IR (ATR): 3115, 1637 (C=0), 1596, 1431, 1367, 1317, 1268, 1180, 1111, $782 \mathrm{~cm}^{-1}$

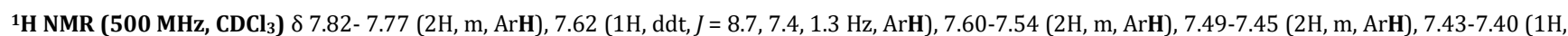
$\mathrm{m} \mathrm{ArH}), 7.39-7.35(1 \mathrm{H}, \mathrm{m}, \mathrm{ArH}), 7.23(1 \mathrm{H}, \mathrm{d}, J=8.0, \mathrm{ArH}), 7.03(1 \mathrm{H}, \mathrm{d}, J=7.5 \mathrm{~Hz}, \mathrm{NH}), 7.01-6.97(1 \mathrm{H}, \mathrm{m}, \mathrm{ArH}), 6.87-6.83(1 \mathrm{H}, \mathrm{m}, \mathrm{ArH}), 5.09(1 \mathrm{H}, \mathrm{ddd}, J=11.8$, 7.5, $4.5 \mathrm{~Hz}, \mathrm{CHNH}), 3.49\left(1 \mathrm{H}, \mathrm{dd}, J=13.7,4.5 \mathrm{~Hz}, \mathrm{CH}_{2} \mathrm{CHNH}\right), 3.20\left(1 \mathrm{H}, \mathrm{dd}, J=13.7,11.5 \mathrm{~Hz}, \mathrm{CH}_{2} \mathrm{CHNH}\right), 2.34(3 \mathrm{H}, \mathrm{s}, \mathrm{CH} 3)$.

${ }^{13} \mathrm{C}$ NMR (125.6 MHz, $\mathbf{C D C l}_{3}$ ) $\delta 199.4(\mathrm{C}=0), 151.0(\mathrm{C}), 139.9(\mathrm{C}), 137.6(\mathrm{C}), 137.3(\mathrm{C}), 136.7(\mathrm{C}), 133.8(\mathrm{CH}), 131.7(\mathrm{CH}), 131.2(\mathrm{CH}), 130.9(2 \times \mathrm{CH}), 130.6$ $(\mathrm{CH}), 128.5(2 \times \mathrm{CH}), 126.4(\mathrm{CH}), 126.1(\mathrm{CH}), 126.0(\mathrm{CH}), 119.4(\mathrm{C}), 119.3(\mathrm{CH}), 58.5(\mathrm{CH}), 37.7\left(\mathrm{CH}_{2}\right), 20.9\left(\mathrm{CH}_{3}\right)$.

HRMS (ESI) Exact mass calculated for $\mathrm{C}_{22} \mathrm{H}_{19} \mathrm{NNaO}_{4} \mathrm{~S}[\mathrm{M}+\mathrm{Na}]^{+}: 416.0927$, found: 416.0928;

The enantiomeric excess was determined by UPC ${ }^{2}$ analysis on a Waters Amy1 column, 100:0 $\mathrm{CO}_{2} / \mathrm{MeOH}$ to $60: 40 \mathrm{CO}_{2} / \mathrm{MeOH}_{\text {for }} 9$ minutes with curve of "6", $\lambda=251 \mathrm{~nm}: \tau_{\text {minor }}=5.70 \mathrm{~min}, \tau_{\text {major }}=5.97 \mathrm{~min}(64 \% \mathrm{ee}) .[\alpha]_{\mathrm{D}}^{28}=+6.2\left(\mathrm{c}=0.76, \mathrm{CHCl}_{3}, 64 \%\right.$ ee $)$.

\section{(R)-(2-((8-methyl-2,2-dioxido-3,4-dihydrobenzo[e][1,2,3]oxathiazin-4-yl)methyl)phenyl)(phenyl)methanone (7c)}

The title compound $\mathbf{7 c}$ was prepared according to the general procedure using $\mathbf{2 d}(9.9 \mathrm{mg}, 50 \mu \mathrm{mol})$ and 2 -methylbenzophenone $\mathbf{1 a}(18 \mu \mathrm{L}, 100 \mu \mathrm{mol})$. The crude product was purified by FC (95:5 hexane/EtOAc) to afford amine 7c (12.4 mg, 63\%, average of two runs) as a white solid. $\mathrm{R}_{\mathrm{f}}=0.31(9: 1$ hexane/EtOAc); $\mathrm{mp}=104-106^{\circ} \mathrm{C}$ (hexane).

IR (ATR): 2921, 1636 (C=0), 1594, 1457, 1370, 1263, 1189, 1151. 1069, $758 \mathrm{~cm}^{-1}$.

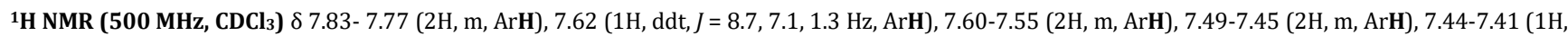
m ArH), 7.39-7.35 (1H, m, ArH), $7.20(1 \mathrm{H}, \mathrm{d}, J=7.6 \mathrm{~Hz}, \mathrm{NH}), 7.18-7.14(1 \mathrm{H}, \mathrm{m}, \mathrm{ArH}), 7.10-7.03$ (2H, m, ArH), 5.12 (1H, ddd, J= 11.8, 7.6, 4.6 Hz, CHNH), 3.49 $\left(1 \mathrm{H}, \mathrm{dd}, J=13.7,4.6 \mathrm{~Hz}, \mathrm{CH}_{2} \mathrm{CHNH}\right), 3.22\left(1 \mathrm{H}, \mathrm{dd}, J=13.7,11.5 \mathrm{~Hz}, \mathrm{CH}_{2} \mathrm{CHNH}\right), 2.30\left(3 \mathrm{H}, \mathrm{s}, \mathrm{CH}_{3}\right)$.

${ }^{13}$ C NMR (125.6 MHz, CDCl$_{3}$ ) $\delta 199.4$ (C=0), 149.7 (C), $137.6(\mathrm{C}), 137.3(\mathrm{C}), 136.8(\mathrm{C}), 133.8(\mathrm{CH}), 131.7$ (CH), $131.2(\mathrm{CH}), 130.9(2 \times \mathrm{CH}), 130.8(\mathrm{CH}), 130.6$ $(\mathrm{CH}), 128.5(2 \times \mathrm{CH}), 128.4(\mathrm{C}), 126.4(\mathrm{CH}), 124.4(\mathrm{CH}), 123.8(\mathrm{CH}), 122.2(\mathrm{C}), 58.8(\mathrm{CH}), 37.9\left(\mathrm{CH}_{2}\right), 15.6\left(\mathrm{CH}_{3}\right)$.

HRMS (ESI) Exact mass calculated for $\mathrm{C}_{22} \mathrm{H}_{19} \mathrm{NNaO}_{4} \mathrm{~S}$ [M+Na]+: 416.0927, found: 416.0911 .

The enantiomeric excess was determined by HPLC analysis on a Daicel IC3 column, 85:15 hexane $/ i$-PrOH, flow rate $=0.8 \mathrm{~mL}, \lambda=254 \mathrm{~nm}: \tau_{\mathrm{major}}=17.95 \mathrm{~min}$, $\tau_{\text {minor }}=21.51 \min (67 \%$ ee $) .[\alpha]_{D^{28}}=-0.38\left(c=0.80, \mathrm{CHCl}_{3}, 67 \%\right.$ ee) . 
(R)-(2-((6-tert-butyl-2,2-dioxido-3,4-dihydrobenzo[e][1,2,3]oxathiazin-4-yl)methyl)phenyl)(phenyl)methanone (7d)

The title compound $\mathbf{7 d}$ was prepared according to the general procedure using $\mathbf{2 e}(12.0 \mathrm{mg}, 50 \mu \mathrm{mol})$ and 2 -methylbenzophenone $\mathbf{1 a}(18 \mu \mathrm{L}, 100 \mu \mathrm{mol})$. The crude product was purified by FC (95:5 hexane/EtOAc) to afford amine 7d (13.1 mg, 60\%, average of two runs) as a white solid. $\mathrm{R}_{\mathrm{f}}=0.38(9: 1 \mathrm{hexane} / \mathrm{EtOAc})$; $\mathrm{mp}=47-49^{\circ} \mathrm{C}$ (hexane).

IR (ATR): 2959, 1646 (C=0), 1489, 1367, 1267, 1186, 1120, 929, 813, $767 \mathrm{~cm}^{-1}$.

1H NMR (500 MHz, CDCl $)$ ) $7.83-7.79$ (2H, m, ArH), 7.65-7.60 (1H, m, ArH), 7.59-7.57 (2H, m, ArH), 7.50-7.45 (2H, m, ArH), 7.43 (1H, dt, J = 7.7, 1.1 Hz, $\operatorname{ArH}), 7.40-7.36(1 \mathrm{H}, \mathrm{m}, \mathrm{ArH}), 7.32(1 \mathrm{H}, \mathrm{ddd}, J=8.6,2.4,0.7, \mathrm{~Hz}, \mathrm{ArH}), 7.30(1 \mathrm{H}, \mathrm{m}, \mathrm{ArH}), 7.06(1 \mathrm{H}, \mathrm{d}, J=7.6, \mathrm{~Hz}, \mathrm{NH}), 6.97(1 \mathrm{H}, \mathrm{d}, J=8.6 \mathrm{~Hz}, \mathrm{ArH}), 5.10(1 \mathrm{H}$, $\mathrm{ddd}, J=12.0,7.6,4.6 \mathrm{~Hz}, \mathrm{CHNH}), 3.52\left(1 \mathrm{H}, \mathrm{dd}, J=13.7,4.7 \mathrm{~Hz}, \mathrm{CH}_{2} \mathrm{CHNH}\right), 3.23\left(1 \mathrm{H}, \mathrm{dd}, J=13.7,11.4 \mathrm{~Hz}, \mathrm{CH} \mathbf{C H N H}_{2} \mathrm{CH}, 30\left(9 \mathrm{H}, \mathrm{s}, \mathrm{C}(\mathrm{CH} 3)_{3}\right)\right.$.

${ }^{13}$ C NMR (125.6 MHz, CDCl 3 ) $\delta 199.4$ (C=0), 148.9 (C), 148.1 (C), 137.7 (C), 137.4 (C), $136.7(\mathrm{C}), 134.8$ (CH), $131.7(\mathrm{CH}), 131.1(\mathrm{CH}), 130.9$ (2 x CH), 130.5 $(\mathrm{CH}), 128.5(2 \times \mathrm{CH}), 126.6(\mathrm{CH}), 126.4(\mathrm{CH}), 122.8(\mathrm{CH}), 121.5(\mathrm{C}), 118.5(\mathrm{CH}), 58.9(\mathrm{CH}), 37.8\left(\mathrm{CH}_{2}\right), 34.5(\mathrm{C}), 31.3\left(3 \times \mathrm{CH}_{3}\right)$.

HRMS (ESI) Exact mass calculated for $\mathrm{C}_{25} \mathrm{H}_{24} \mathrm{NO}_{4} \mathrm{~S}[\mathrm{M}+\mathrm{H}]^{+}: 434.1432$, found: 434.1421.

The enantiomeric excess was determined by UPC 2 analysis on a Waters Amy1 column, 100:0 $\mathrm{CO}_{2} / \mathrm{EtOH}$ to $60: 40 \mathrm{CO} / \mathrm{EtOH}_{2}$ for 9 minutes with curve of "6", $\lambda=260 \mathrm{~nm}: \tau_{\text {minor }}=4.92 \mathrm{~min}, \tau_{\text {major }}=5.20 \mathrm{~min}(62 \%$ ee $) .[\alpha]_{\mathrm{D}}{ }^{28}=+12.3\left(\mathrm{c}=0.62, \mathrm{CHCl}_{3}, 62 \%\right.$ ee $)$.

\section{(R)-(2-((7-methoxy-2,2-dioxido-3,4-dihydrobenzo[e][1,2,3]oxathiazin-4-yl)methyl)phenyl)(phenyl)methanone (7e)}

The title compound $\mathbf{7 e}$ was prepared according to the general procedure (but with a reaction time of 72 hours rather than 48 hours) using $\mathbf{2 f}(10.7$ mg, 50 $\mu \mathrm{mol})$ and 2-methylbenzophenone 1a $(18 \mu \mathrm{L}, 100 \mu \mathrm{mol})$. The crude product was purified by FC (95:5 hexane/EtOAc) to afford amine 7e (7.8 mg, 38\%, average of two runs) as a white solid. $\mathrm{R}_{\mathrm{f}}=0.24$ (9:1 hexane/EtOAc); $\mathrm{mp}=59-61^{\circ} \mathrm{C}$ (hexane).

IR (ATR): 2922, 1636 (C=0), 1575, 1505, 1372, 1268, 1184, 1152, 1092, $746 \mathrm{~cm}^{-1}$.

1H NMR (500 MHz, CDCl $)$ ) $8.84-7.79$ (2H, m, ArH), 7.69-7.56 (3H, m, ArH), 7.53-7.47 (2H, m, ArH), 7.47-7.43 (1H, m, ArH), 7.42-7.37 (1H, m ArH), 7.29$7.26(1 \mathrm{H}, \mathrm{m}, \mathrm{ArH}), 7.08(1 \mathrm{H}, \mathrm{d}, J=7.6 \mathrm{~Hz} \mathrm{NH}), 6.77(1 \mathrm{H}, \mathrm{dd}, J=8.7,2.6 \mathrm{~Hz}), 5.59(1 \mathrm{H}, \mathrm{d}, J=2.6 \mathrm{~Hz}), 5.09(1 \mathrm{H}, \mathrm{ddd}, J=11.8,7.6,4.5 \mathrm{~Hz}, \mathrm{CHNH}), 3.82(3 \mathrm{H}, \mathrm{s} \mathrm{CH} 3)$, $3.49\left(1 \mathrm{H}, \mathrm{dd}, J=13.6,4.5 \mathrm{~Hz}, \mathrm{CH}_{2} \mathrm{CHNH}\right), 3.22\left(1 \mathrm{H}, \mathrm{dd}, J=13.6,11.4 \mathrm{~Hz}, \mathrm{CH}_{2} \mathrm{CHNH}\right)$.

${ }^{13}$ C NMR (125.6 MHz, CDCl $) \delta 199.4$ (C=0), 148.9 (C), 148.1(C), 137.7 (C), 137.3 (C), 136.7 (C), $133.8(\mathrm{CH}), 131.7$ (CH), $131.1(\mathrm{CH}), 130.9$ (2 x CH), 130.5 (C), $128.5(2 \times \mathrm{CH}), 126.6(\mathrm{CH}), 126.4(\mathrm{CH}), 122.8(\mathrm{CH}), 121.5(\mathrm{C}), 118.5(\mathrm{CH}), 58.9(\mathrm{CH}), 37.7\left(\mathrm{CH}_{2}\right), 31.3\left(\mathrm{CH}_{3}\right)$.

HRMS (ESI) Exact mass calculated for $\mathrm{C}_{22} \mathrm{H}_{19} \mathrm{NNaO}_{5} \mathrm{~S}[\mathrm{M}+\mathrm{Na}]^{+}: 432.0876$, found: 432.0881

The enantiomeric excess was determined by UPC 2 analysis on a Waters Amy1 column, 100:0 $\mathrm{CO}_{2} / \mathrm{EtOH}$ to $60: 40 \mathrm{CO} / \mathrm{EtOH}_{2}$ for 9 minutes with curve of "6", $\lambda=250 \mathrm{~nm}: \tau_{\text {minor }}=5.77 \mathrm{~min}, \tau_{\text {major }}=6.03 \mathrm{~min}(69 \%$ ee $) \cdot[\alpha]_{\mathrm{D}}{ }^{28}=+5.7\left(\mathrm{c}=0.50, \mathrm{CHCl}_{3}, 69 \%\right.$ ee $)$.

\section{(R)-(2-((6-fluoro-2,2-dioxido-3,4-dihydrobenzo[e][1,2,3]oxathiazin-4-yl)methyl)phenyl)(phenyl)methanone (7f)}

The title compound $\mathbf{7 f}$ was prepared according to the general procedure (but with a reaction time of 72 hours rather than 48 hours) using $2 \mathbf{g}$ ( 10.1 mg, 50 $\mu \mathrm{mol})$ and 2-methylbenzophenone $\mathbf{1 a}(18 \mu \mathrm{L}, 100 \mu \mathrm{mol})$. The crude product was purified by FC (95:5 hexane/EtOAc) to afford amine $\mathbf{7 f}(8.5 \mathrm{mg}, 43 \%$, average of two runs) as a white solid. $\mathrm{R}_{\mathrm{f}}=0.31$ (9:1 hexane/EtOAc); $\mathrm{mp}=55-57^{\circ} \mathrm{C}$ (hexane).

IR (ATR): 3063, 1935 (C=0), 1487, 1418, 1373, 1257, 1190, 1157, 929, $763 \mathrm{~cm}^{-1}$.

${ }^{1} \mathbf{H}$ NMR (500 MHz, CDCl $)$ ) 7.87-7.74 (2H, m, ArH), 7.66-7-55 (3H, m, ArH), 7.52-7.46 (2H, m, ArH), 7.46-7.42 (1H, m, ArH), 7.42-7.37 (1H, m ArH), 7.18 $(1 \mathrm{H}, \mathrm{d}, J=7.6 \mathrm{~Hz}, \mathrm{NH}), 7.12-7.07(1 \mathrm{H}, \mathrm{m}, \mathrm{ArH}), 7.06-6.99(1 \mathrm{H}, \mathrm{m} \mathrm{ArH}), 7.01-6.97(1 \mathrm{H}, \mathrm{m}, \mathrm{ArH}), 5.12(1 \mathrm{H}, \mathrm{ddd}, J=11.9,7.6,4.6 \mathrm{~Hz}, \mathrm{CHNH}), 3.45(1 \mathrm{H}, \mathrm{dd}, J=$ 13.6, $\left.4.6 \mathrm{~Hz}, \mathrm{CH}_{2} \mathrm{CHNH}\right), 3.24\left(1 \mathrm{H}, \mathrm{dd}, J=13.6,11.5 \mathrm{~Hz}, \mathrm{CH}_{2} \mathrm{CHNH}\right)$.

${ }^{13}$ C NMR (125.6 MHz, $\mathbf{C D C l}_{3}$ ) $\delta 196.7$ (C=0), 156.4 (d, J = 245.0 Hz, C), 144.3 (d, J = 2.5 Hz, C), 134.7 (C), 134.4 (C), 133.5 (C), 131.1 (CH), 129.1 (CH), 128.3 $(\mathrm{CH}), 128.1(2 \times \mathrm{CH}), 128.0(\mathrm{CH}), 126.7(2 \times \mathrm{CH}), 123.8(\mathrm{CH}), 121.1(\mathrm{~d}, J=6.9 \mathrm{~Hz}, \mathrm{C}), 117.7(\mathrm{~d}, \mathrm{~J}=8.3 \mathrm{~Hz}, \mathrm{CH}), 113.6(\mathrm{~d}, J=23.6 \mathrm{~Hz}, \mathrm{CH}), 110,2(\mathrm{~d}, J=24.9 \mathrm{~Hz}$, $\mathrm{CH}), 55.7(\mathrm{~d}, J=1.7 \mathrm{~Hz}, \mathrm{CH}), 34.7\left(\mathrm{CH}_{2}\right)$.

${ }^{19}$ F NMR (376 MHz, $\left.\mathbf{C D C l}_{3}\right) \delta-116.3(\mathrm{dt}, J=8.9,6.3 \mathrm{~Hz}, \mathrm{~F})$.

HRMS (ESI) Exact mass calculated for $\mathrm{C}_{21} \mathrm{H}_{16} \mathrm{FNNaO}_{4} \mathrm{~S}$ [M+Na] ${ }^{+}: 420.0676$, found: 420.0682 .

The enantiomeric excess was determined by UPC 2 analysis on a Waters Amy1 column, 100:0 $\mathrm{CO}_{2} / \mathrm{EtOH}$ to $60: 40 \mathrm{CO} / \mathrm{EtOH}_{2}$ for 9 minutes with curve of " 6 ", $\lambda=251 \mathrm{~nm}: \tau_{\text {minor }}=4.95 \mathrm{~min}, \tau_{\text {major }}=5.21 \mathrm{~min}(54 \%$ ee $) \cdot[\alpha]_{\mathrm{D}}{ }^{28}=+0.65\left(\mathrm{c}=0.59, \mathrm{CHCl}_{3}, 54 \%\right.$ ee $)$.

\section{(R)-(2-((6-chloro-2,2-dioxido-3,4-dihydrobenzo[e][1,2,3]oxathiazin-4-yl)methyl)phenyl)(phenyl)methanone (7g)}

The title compound $\mathbf{7 g}$ was prepared according to the general procedure using $\mathbf{2 h}(10.9 \mathrm{mg}, 50 \mu \mathrm{mol})$ and 2 -methylbenzophenone $\mathbf{1 a}(18 \mu \mathrm{L}, 100 \mu \mathrm{mol})$. The crude product was purified by FC (95:5 hexane/EtOAc) to afford amine $7 \mathbf{g}\left(14.5 \mathrm{mg}, 70 \%\right.$, average of two runs) as a white solid. $\mathrm{R}_{\mathrm{f}}=0.32$ ( $\left.9: 1 \mathrm{hexane} / \mathrm{EtOAc}\right)$; $\mathrm{mp}=63-65^{\circ} \mathrm{C}$ (hexane).

IR (ATR): 2922, 1645 (C=0), 1539, 1473, 1373, 1259, 1187, 1165, 1113, $769 \mathrm{~cm}^{-1}$.

${ }^{1} \mathbf{H}$ NMR (500 MHz, CDCl $)$ ) 7.82- 7.77 (2H, m, ArH), 7.66-7.59 (2H, m, ArH), 7.58-7.55 (1H, m, ArH), 7.51-7.46 (2H, m, ArH), 7.45-7.43 (1H, m ArH), 7.42$7.35(1 \mathrm{H}, \mathrm{m}, \mathrm{ArH}), 7.31-7.27(2 \mathrm{H}, \mathrm{ArH}), 7.00(2 \mathrm{H}, \mathrm{d}, J=8.8 \mathrm{~Hz}), 5.11(1 \mathrm{H}, \mathrm{ddd}, J=12.0,7.7,4.7 \mathrm{~Hz}, \mathrm{CHNH}), 3.48(1 \mathrm{H}, \mathrm{dd}, J=13.6,4.7 \mathrm{~Hz}, \mathrm{CH} 2 \mathrm{CHNH}), 3.22(1 \mathrm{H}$, $\left.\mathrm{dd}, J=13.6,11.6 \mathrm{~Hz}, \mathrm{CH}_{2} \mathrm{CHNH}\right)$.

${ }^{13}$ C NMR (125.6 MHz, $\mathbf{C D C l}_{3}$ ) $\delta 199.6$ (C=0), $149.8(\mathrm{C}), 137.5(\mathrm{C}), 137.2(\mathrm{C}), 136.2(\mathrm{C}), 134.0(\mathrm{CH}), 131.9(\mathrm{CH}), 131.1(\mathrm{CH}), 131.0(2 \times \mathrm{CH}), 130.8(\mathrm{CH}), 130.3$ (C), $129.5(\mathrm{CH}), 128.5(2 \times \mathrm{CH}), 126.6(\mathrm{CH}), 126.3(\mathrm{CH}), 124.0(\mathrm{C}), 120.5(\mathrm{CH}), 58.4(\mathrm{CH}), 37.5\left(\mathrm{CH}_{2}\right)$.

HRMS (ESI) Exact mass calculated for $\mathrm{C}_{21} \mathrm{H}_{16} \mathrm{ClNNaO}_{4} \mathrm{~S}[\mathrm{M}+\mathrm{Na}]^{+}: 436.0381$, found: 436.0394 .

The enantiomeric excess was determined by UPC 2 analysis on a Waters Amy1 column, 100:0 $\mathrm{CO}_{2} / \mathrm{EtOH}$ to $60: 40 \mathrm{CO} / 2 \mathrm{EtOH}$ for 9 minutes with curve of "6", $\lambda=251 \mathrm{~nm}: \tau_{\text {minor }}=5.28 \mathrm{~min}, \tau_{\text {major }}=5.63 \mathrm{~min}(47 \% \mathrm{ee}) \cdot[\alpha]_{\mathrm{D}}{ }^{28}=+5.8\left(\mathrm{c}=0.89, \mathrm{CHCl}_{3}, 47 \%\right.$ ee $)$. 


\section{(R)-(2-((7-bromo-2,2-dioxido-3,4-dihydrobenzo[e][1,2,3]oxathiazin-4-yl)methyl)phenyl)(phenyl)methanone (7h)}

The title compound $\mathbf{7 h}$ was prepared according to the general procedure using $\mathbf{2 i}(13.0 \mathrm{mg}, 50 \mu \mathrm{mol})$ and 2 -methylbenzophenone $\mathbf{1 a}(18 \mu \mathrm{L}, 100 \mu \mathrm{mol})$. The crude product was purified by FC (95:5 hexane/EtOAc) to afford amine $7 \mathbf{h}\left(17.2 \mathrm{mg}, 75 \%\right.$, average of two runs) as a white solid. $\mathrm{R}_{\mathrm{f}}=0.33(9: 1 \mathrm{hexane} / \mathrm{EtOAc})$; $\mathrm{mp}=119-121^{\circ} \mathrm{C}$ (hexane).

IR (ATR): 3063, 1635 (C=0), 1595, 1477, 1363, 1268, 1184, 1122, 907, $763 \mathrm{~cm}^{-1}$.

1H NMR (500 MHz, CDCl $)$ ) 7.82- 7.77 (2H, m, ArH), 7.63 (1H, ddt, J = 8.7, 7.1, 1.3 Hz, ArH), 7.61-7.57 (1H, m, ArH), 7.57-7.53 (1H, m, ArH), 7.50-7.45 (2H, $\mathrm{m} \mathrm{ArH}), 7.43(1 \mathrm{H}, \mathrm{dd}, J=7.7,1.5 \mathrm{~Hz}, \operatorname{ArH}), 7.40-7.36(1 \mathrm{H}, \mathrm{m}, \mathrm{ArH}), 7.32(1 \mathrm{H}, \mathrm{dd}, J=8.4,2.0, \mathrm{~Hz}, \operatorname{ArH}), 7.25-7.22(1 \mathrm{H}, \mathrm{m}, \operatorname{ArH}), 7.21(1 \mathrm{H}, \mathrm{d}, J=2.0, \mathrm{~Hz}, \mathrm{NH})$, $6.97(1 \mathrm{H}, \mathrm{d}, J=8.6 \mathrm{~Hz}, \mathrm{ArH}), 5.08(1 \mathrm{H}, \mathrm{ddd}, J=12.0,7.6,4.6 \mathrm{~Hz}, \mathrm{CHNH}), 3.47\left(1 \mathrm{H}, \mathrm{dd}, J=13.7,4.6 \mathrm{~Hz}, \mathrm{CH} \mathrm{CHNH}, 3.21\left(1 \mathrm{H}, \mathrm{dd}, J=13.7,11.5 \mathrm{~Hz}, \mathrm{CH}{ }_{2} \mathrm{CHNH}\right)\right.$.

${ }^{13}$ C NMR (125.6 MHz, CDCl 3$) \delta 199.5$ (C=0), 151.5 (C), 137.5 (C), 137.2 (C), 136.3 (C), $133.9(\mathrm{CH}), 131.9$ (CH), $131.2(\mathrm{CH}), 130.9$ (2 x CH), 130.8 (CH), 128.5 $(2 \times \mathrm{CH}), 128.3(\mathrm{CH}), 127.6(\mathrm{CH}), 126.6(\mathrm{CH}), 122.2(\mathrm{CH}), 122.2(\mathrm{C}), 121.5(\mathrm{C}), 58.4(\mathrm{CH}), 37.6\left(\mathrm{CH}_{2}\right)$.

HRMS (ESI) Exact mass calculated for $\mathrm{C}_{21} \mathrm{H}_{16} \mathrm{BrNNaO}_{4} \mathrm{~S}[\mathrm{M}+\mathrm{Na}]^{+}: 479.9876$, found: 479.9875.

The enantiomeric excess was determined by UPC 2 analysis on a Waters Cel1 column, 100:0 $\mathrm{CO}_{2} / \mathrm{MeOH}$ to $60: 40 \mathrm{CO}_{2} / \mathrm{MeOH}_{\text {for }} 9$ minutes with curve of "6", $\lambda=250 \mathrm{~nm}: \tau_{\text {minor }}=4.68 \mathrm{~min}, \tau_{\text {major }}=4.79 \mathrm{~min}(53 \%$ ee $) \cdot[\alpha]_{\mathrm{D}}{ }^{28}=+1.8\left(\mathrm{c}=0.81, \mathrm{CHCl}_{3}, 53 \%\right.$ ee $)$.

\section{Acknowledgment}

Financial support was provided by the ICIQ Foundation, MINECO (project CTQ2013-45938-P and Severo Ochoa Excellence Accreditation 2014-2018, SEV2013-0319), the AGAUR (2014 SGR 1059), and the European Research Council (ERC 278541 - ORGA-NAUT). H.H. thanks the Marie Curie COFUND action (2015-1-ICIQ-IPMP) for a postdoctoral fellowship. G.M. thanks ICIQ-LMU (SEV-2013-0319) for a predoctoral fellowship. The authors thank Dr. Luca Dell'Amico for preliminary investigations and insightful discussions, and Sara Cuadros and Dr. Javier Perez for assistance with the transient absorption spectroscopic experiments.

\section{Supporting Information}

YES (this text will be updated with links prior to publication)

\section{Primary Data}

NO (this text will be deleted prior to publication)

\section{References}

(1) Yang, N. C.; Rivas, C. J. Am. Chem. Soc. 1961, 83, 2213-2213.

(2) Sammes, P. G. Tetrahedron 1976, 32, 405-422.

(3) a) Klán, P.; Wirz, J.; Gudmundsdottir, A. Photoenolization and its applications. In CRC Handbook of Organic Photochemistry and Photobiology, Griesbeck A., CRC Press, $3^{\text {rd }}$ ed., 2012 Chapter 26, pp. 627-651; b) Block, E.; Stevenson, R. J. Chem. Soc. Perkin I. 1973, 308-313..

(4) a) Haag, R. ; Wirz, J.; Wagner, P. J. Helv. Chim. Acta 1977, 60, 2595-2607; b) Scaiano, J. C. Acc. Chem. Res. 1982, 15, 252-258; c) Wagner, P. J.; Chen, C. P. J. Am. Chem Soc. 1976, 98, 239-241; d) Das, P. K.; Scaiano, J. C. J. Photochem., 1980, 12, 85-90.

(5) For selected examples, see: a) Nicolaou, K. C.; Gray, D.; Tae, J. Angew. Chem. Int. Ed. 2001, 40, 3675-3678; b) Nicolaou, K. C.; Gray, D.; Tae, J. Angew. Chem. Int. Ed. 2001, 40, 3679-3683; c) Nicolaou, K. C.; Gray, D. J. Am. Chem. Soc. 2004, 126, 613-627; d) Charlton, J. L.; Koh, K. J. Org. Chem. 1992, 57, 1514-1516.

(6) Grosch, B.; Orlebar, C. N.; Herdtweck, E.; Massa, W.; Bach, T. Angew. Chem. Int. Ed. 2003, 42, 3693-3696.

(7) Dell'Amico, L.; Vega-Peñaloza, A.; Cuadros, S.; Melchiorre, P. Angew. Chem. Int. Ed. 2016, 55, 3313-3317.

(8) a) Dalko, P. I.; Ed., Comprehensive Enantioselective Organocatalysis: Catalysts, Reactions, and Applications (Wiley-VCH, Weinheim, 2013); b) MacMillan, D. W. C. Nature 2008, 455, 304-308.

(9) Merino, P.; Delso, I.; Tejero, T.; Roca-Lopez, D.; Isasi, A.; Matute, R. Curr. Org. Chem. 2011, 15, 2184-2209

(10) For overviews, see: a) Jørgensen, K. A. Angew. Chem. Int. Ed. 2000, 39, 3558-3588. b) Tietze, L. F.; Kettschau, G. Top. Curr. Chem. 2008, $189,1-120$.

(11) For reviews, see: a) Adair, G.; Mukherjee, S.; List, B. Aldrichimica Acta 2008, 41, 31-39; b) Connon, S. J. Chem. Comm. 2008, $2499-2510$.

(12) For early examples of using cyclic aldimine 2a as a prochiral electrophile, see: a) Luo, Y.; Carnell, A. J.; Lam, H. W. Angew. Chem. Int. Ed. 2012, 51, 67626766; b) Luo. Y.; Hepburn, H. B.; Chotsaeng, N.; Lam, H. W. Angew. Chem. Int. Ed. 2012, 51, 8309-8313. For an early example of using the corresponding ketimine, see: c) Nishimura, T.; Noishiki, A.; Chit Tsui, G.; Hayashi, T. J. Am. Chem. Soc. 2012, 134, 5056-5059.

(13) For an example of imine 2a participating in an enantioselective catalytic hetero-Diels-Alder reaction, see: Liu, Y.; Kang, T.-R.; Liu, Q.-Z.; Chen, L.-M.; Wang, Y.-C.; Liu, J.; Xie, Y.-M.; Yang, J.-L.; He, L. Org. Lett. 2013, 15, 6090-6093.

(14) Amberg, W.; Bennani, Y. L.; Chadha, R. K.; Crispino, G. A.; Davies, W. D.; Hartung, J.; Jeong, K. S.; Ogino, Y.; Shibata, T.; Sharpless, K. B. J. Org. Chem. 1993, $58,844-849$.

(15) In addition to the rate acceleration provided by catalyst $6 \mathbf{6}$, the observed level of stereoselectivity can be rationalized on the basis of solubility issues. Indeed, under the reaction conditions (initial concentration of cyclic imine $\mathbf{2 a}$ is $0.025 \mathrm{M}$ in cyclohexane) the substrate $\mathbf{2 a}$ is only partially soluble. This condition secures a low amount of electrophile in the organic solvent interacting with the (fully soluble) chiral catalyst 6 a.

(16) In analogy with our precedent study on the stereoselective trapping of the photoenol A with maleimides promoted by the cinchona-derived catalyst 5c (Ref. 7), we evaluated the possibility for the chiral amine 6a to attenuate the rate of the racemic background process by reducing the concentration of $\mathbf{A}$ in solution. Flash photolysis quenching studies of the transient photoenol $\mathbf{A}$ at the millisecond resolution, detailed in the Supplementary Information, established that the amine 6 a does not influence the formation of $\mathbf{A}$.

(17) Crystallographic data for compound 3e has been deposited with the Cambridge Crystallographic Data Centre, accession numbers CCDC 1497811. Other entries were assigned by analogy. 
(18) Masuda, Y.; Ishida, N.; Murakami, M. J. Am. Chem. Soc. 2015, 137, 14063-14066.

(19) Previous reports, which have used (DHQ) 2 PHAL 6a to catalyze enantioselective processes, invoked hydrogen bonding interactions (through the phthalazine moiety) and $\pi-\pi$ interactions (via the quinoline moiety) with the substrates to rationalize the high stereocontrol, see: a) Whitehead, D. C.; Yousefi, R.; Jaganathan, A.; Borhan, B. J. Am. Chem. Soc. 2010, 132, 3298-3300; b) Yousefi, R.; Whitehead, D. C.; Mueller, J. M.; Staples, R. J.; Borhan, B. Org. Lett. 2011, 13, 608-611; c) Nicolaou, K. C.; Simmons, N. L.; Ying, Y.; Heretsch, P. M.; Chen, J. S. J. Am. Chem. Soc. 2011, 133, 8134-8137; d) Wilking, M.; Mück-Lichtenfeld, M.; Daniliuc, C. G.; Hennecke, U. J. Am. Chem. Soc. 2013, 135, 8133-8136; e) Zhang, W.; Liu, N.; Schienebeck, C. M.; Zhou, X.; Izhar, I. I.; Guzei, I. A.; Tang, W. Chem. Sci. 2013, 4, 2652-2656; f) Yin, Q.; W. S.-G.; Liang, X.-W.; Gao, D.-W.; Zheng, J.; You, S.-L. Chem. Sci. 2015, 6, 41794183; g) Zhang, T.; Qiao, Z.; Wang, Y.; Zhong, N.; Liu, L.; Wang, D.; Chen, Y.-J. Chem. Commun. 2013, 49, 1636-1638; h) Di Iorio, N.; Champavert, F.; Erice, A.; Righi, P.; Mazzanti, A.; Bencivenni, G. Tetrahedron, 2016, 72, 5191-5201.

(20) Litvinas, N. D.; Brodsky, B. H.; Du Bois, J. Angew. Chem. Int. Ed. 2009, 48, 4513-4516. 